\title{
PAMELA: A Usefful Tool for the Study of Leaky-Wave Modes in Strip-Loaded Open Dielectric Waveguides
}

\author{
Jose Luis Gómez-Tornero, David Cañete Rebenaque, Fernando Quesada-Pereira, Juan Pascual Martinez, and \\ Alejandro Álvarez-Melcón \\ Information and Communications Department, Technical University of Cartagena \\ Antiguo Hospital de Marina. 30202 Cartagena, Murcia, Spain \\ Tel: +0034968326531, +0034968325315, +0034968 338865; Fax; +0034968 325973; \\ E-mail: josel.gomez@upct.es, david.canete@upct.es, fernando.quesada@upct.es, juan.pascual@upct.es, \\ alejandro.alvarez@upct.es
}

\begin{abstract}
A software tool is presented to help in the teaching of the electromagnetic theory of open dielectric waveguides. The study of the modal spectrum of canonical closed waveguides is a basic topic in any course of electromagnetics or microwave engineering. However, surface-wave modes and leaky-wave modes in open waveguides are not yet so well known in the academic environment. Nevertheless, this study is necessary, since novel open dielectric waveguides have been proposed for millimeter-wave and optical electronics in order to reduce the conductor losses. Therefore, the comprehension of the phenomenon of leaky-wave modes and behavior is indispensable for any future microwave engineer, who will have to work with the new millimeter-wave and optical technologies. This program constitutes the first tool explicitly conceived to help the teaching of the working principles of open dielectric guides loaded with printed circuits, and the associated practical leakywave antennas (LWAs). The code can be a very good supplement to understand how practical leaky-wave antennas work, after a basic theoretical understanding of leaky waves has been first obtained. From the appearance of leaky-wave modes in open waveguides to the design of a backward-to-forward leaky-wave antenna, some simple and very clarifying step-by-step exercises are presented to illustrate these not-so-well-known concepts. Although the program refers to a specific type of open dielectric guides (laterally-shielded, top-open, stub-loaded, rectangular dielectric waveguides with planar perturbations), the results are applicable to any open dielectric guide, helping to teach the basic theory and topics relating to leaky- and surfacewave modes in uniform and periodic structures.
\end{abstract}

Keywords: Dielectric loaded waveguides; nonhomogeneously loaded waveguides; nonradiative dielectric waveguides; printed circuits; waveguide antennas; leaky waves; leaky wave antennas; computer aided engineering; computer aided instruction; electrical engineering education

\section{Introduction}

$\mathbf{T}$ The topic of waveguides is one of the basic chapters in any course of electromagnetics. Rectangular, circular, and coaxial waveguides can be analytically studied due to their canonical geometries, and their constitutive equations can be easily derived from Maxwell's equations [1,2]. The set of permitted modes for each waveguide is obtained from these equations, and from this arises its modal spectrum. Many important and basic aspects such as the cutoff frequency, the field pattern inside the waveguide, and the frequency dispersion of each mode - need to be taught to an electric engineer in order to understand the working principles of waveguide propagation. More-complicated waveguides - many of which have open boundaries and planar metallizations - have been proposed for millimeter-wave and optical electronics, in order to reduce the conductor losses while maintaining a simple geometry [3]. These novel waveguides cannot be analytically analyzed, and numerical techniques must be developed for their study [3]. Radiation from these open waveguides can occur, being an unwanted phenomenon, since it creates crosstalk and radiation losses $[4,5]$. Moreover, radiation from open waveguides has been extensively used in order to create novel leaky-wave antennas for the millimeter-wave band [6]. In any case, it is necessary to understand which conditions cause a given mode of an open waveguide to radiate.

Leaky-wave modes are part of the modal spectrum of open waveguides, and they can solely describe the radiated field pattern of a leaky-wave antenna under some conditions (basically, when direct radiation from the source of the open waveguide - continuous spectrum - is avoided). Therefore, the propagation and radiation characteristics of a given leaky-wave mode (maximum radiation direction, beamwidth, polarization, frequency bandwidth, etc.) must be known and controlled in order to design a leaky-wave antenna (LWA). There are basically two types of leaky-wave antennas that provide radiation from open waveguides by introducing perturbations, namely uniform and periodic leaky-wave antennas [6]. Figure 1 shows two uniformly loaded dielectric waveguides and one periodically perturbed guide (Figure la corresponds to a uniform slotted structure, Figure $1 \mathrm{~b}$ is a uniform striploaded guide, and Figure 1c shows a periodic strip-loaded guide). 

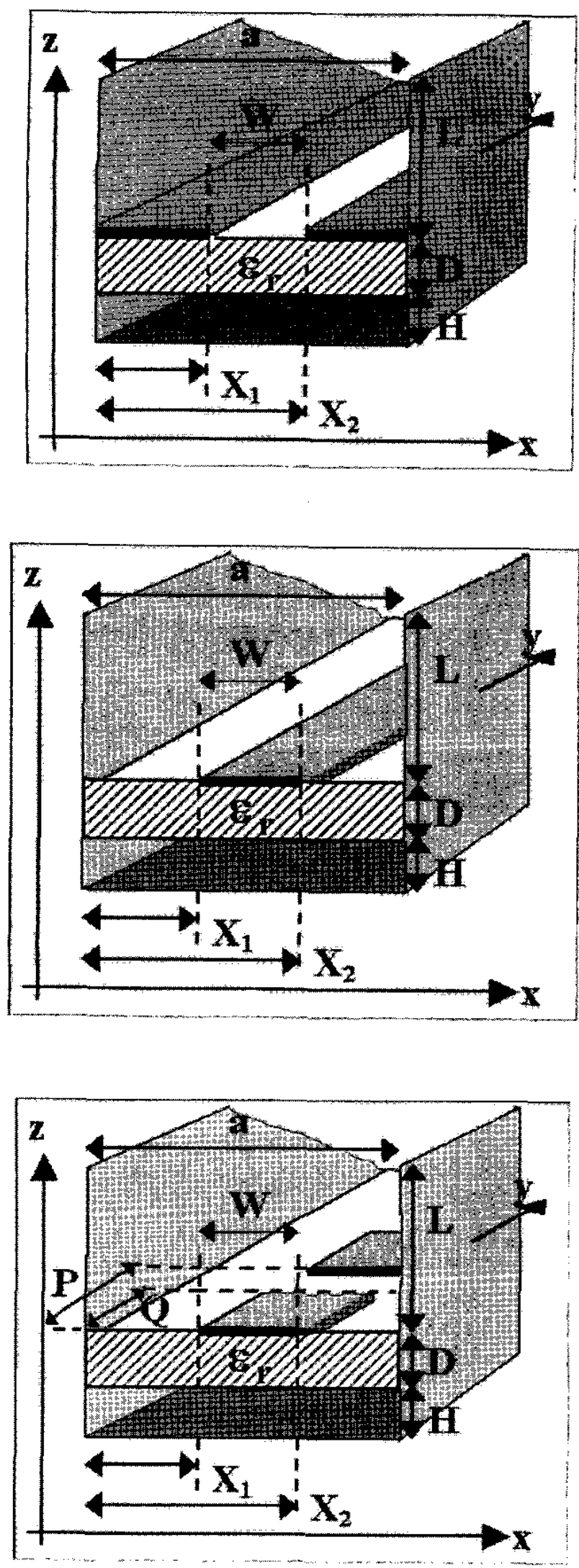

Figure 1. Laterally-shielded open dielectric waveguides with planar perturbations: ( $a$, top) a uniform slotted structure; (b, middle) a uniform strip-loaded guide; and (c, bottom) a periodic strip-loaded guide.
The radiation mechanisms in each type of structure (uniform or periodic) have some points in common and some differences.

This paper describes some simple and illustrative exercises that can be done with the help of a software tool and associated graphical user interface (GUI) environment, in order to understand the theory behind the propagation and radiation of leaky waves in such laterally-shielded open dielectric guides, perturbed with planar circuitry. Leaky-wave modes have been known for the last four decades $[7,8]$, and many articles and works have been published, studying their behavior in different open waveguides, and their utility for conceiving novel leaky-wave antennas $[9,10]$. The impact of planar technology and new open dielectric waveguides for the millimeter and optical ranges in the last two decades confers a more practical and urgent need for teaching future engineers the basic concepts concerning leaky-wave modes and their applications [11]. However, the study of leaky-wave modes has not yet been introduced in electromagnetism education for future microwave engineers, probably due to the absence of any commercial software capable of analyzing such modes in a student-friendly way. For this purpose, a novel interactive environment has been created for the analysis of leaky-wave modes in some types of laterally-shielded open waveguides, such as those shown in Figure 1. The program has been named PAMELA (Program for the Modal Analysis of Laterally Shielded Structures, in Spanish). It has been developed using $M A T L A B^{\mathrm{TM}} 6.5$, in order to take advantage of its graphical facilities. Due to the highly analytical and matrix nature of the implemented method $[12,13]$, the computations are fast enough to permit interactive work, obtaining visual results in rea] time.

The main objective of this paper is to show how this program can help to teach some advanced concepts relating to the nature of radiation from open dielectric waveguides loaded with uniform and periodic printed circuits. After the students have become familiar with the basic concepts of leaky waves on structures, which can be studied theoretically (for instance, with simple dielectric layers), this code can be a good supplement to numerically analyze a morecomplicated (but also more-practical) type of leaky-wave-antenna structure. The GUI associated with the software is described in Section 2, while the next sections present some simple but very illustrative exercises to aid in understanding the leakage phenomenon in the proposed structures. Section 3 presents the study of a completely shielded strip-loaded uniform dielectric waveguide. This first exercise helps in understanding the working mechanism of the program, and also aids in easier understanding of what happens to the modes of a closed waveguide when it is opened. Section 4 extends in the study of surface and leaky-wave modes in open dielectric guides, while the frequency response of leaky-wave modes and the transition to surface waves is studied in detail in Section 5. Section 6 shows how some types of leaky-wave modes can be used to conceive leaky-wave antennas (LWA). The beandirection frequency-scanning capability of leaky-wave antennas, and the problem of the variation of the beamwidth with frequency, are also studied in this section. Section 7 presents the study of a periodic structure, introducing space hamonics theory and the phenomenon of backward leaky-wave radiation. Finally, Section 8 shows how to use this program to analyze and design a practical backward-to-forward leaky-wave antenna. The conclusion section summarizes all the concepts that can be trained using this program. These concepts can be learned by the student thanks to the combination of the mathematical expressions and the graphical results presented in this paper. 


\section{Program Description}

A GUI (graphical user interface) has been developed to combine, in a single software tool, all the elements needed to obtain the modal spectrum of laterally-shielded open waveguides, such as those shown in Figure 1. The main window of the program is shown in Figure 3. The basic theory underlying the study of closed waveguides [2] must be well known to the student. The first purpose is to obtain the propagation constants in the longitudinat direction of the waveguide ( $k_{y}$, according to the reference axes of 、 Figure 1) for the permitted modes. For the case of leaky-wave modes, the propagation constant is complex, with a real part that is the longitudinal phase constant $\left(\beta_{y}\right)$ and an imaginary part, also called the attenuation constant, $\alpha_{y}$

$$
k_{y}=\beta_{y}-j \alpha_{y}=k_{0} \sqrt{\varepsilon_{r e f f}}\left[\mathrm{~m}^{-1}\right]
$$

where $k_{0}$ is the free-space wavenumber, and $\varepsilon_{r e f f}$ is the effective relative constant, which, in general, can have a complex value. The complex nature of the longitudinal propagation constant is due to the decrease of the amplitude of the leaky-wave mode as it propagates through the open waveguide, as can be inferred from the inverse Fourier transform of a sinusoidal time-varying field:

$$
\begin{aligned}
& \tilde{A}\left(k_{y}\right)=e^{-j k_{y} y}=e^{-j\left(\beta_{y}-j \alpha_{y}\right) y}=e^{-j \beta_{y} y} e^{-\alpha_{y} y}, \\
& A\left(\omega_{0} t\right)=\Re R\left\{\tilde{A}\left(k_{y}\right) e^{j \omega_{0} t}\right\}=e^{-\alpha_{y} y} \cos \left(\omega_{0} t-\beta_{y} y\right) .
\end{aligned}
$$

This attenuation might be caused by radiation losses. That is, the mode suffers leakage of power as it propagates, radiating at a given angle, $\theta_{m}$, and it is therefore called a leaky-wave mode. Figure 2 illustrates this phenomenon.

Figure 3 shows the main window of PAMELA, a program specifically developed to study and teach leaky-wave modes in a visual and interactive way.

The user must first choose the type of structure to be studied (strip, slot, no planar metallization), and must introduce the values for its geometrical parameters (according to Figure 1). This is done using the Input parameter window, which appears when the button indicated with the number 1 (Input Parameters) in Figure 3 is pressed. The top-left region of the main window shows a figure of the type of structure that is being analyzed (strip/slot, closed/open waveguide, uniform/periodic), together with its constitutive dimensions, as shown in Figure 1. Loading and saving controls permit storing the parameters introduced in the main window (the geometrical parameters of the structure and the numerical parameters for the analysis) in a text file, and allow recovering them in a new session. The numerical method used for the electromagnetic analysis is based on the Method of Moments (MoM), and the formulation can be found in $[12,13]$. The kernel of the program computes the determinant of the MoM matrix (det $P$ ). This determinant is a function that depends on the geometry, the frequency, and the value of $k_{y}$. A given $k_{y}$ value will be a solution of Maxwell's equations for a given geometry, $G$, and frequency, $f$, if the following condition is satisfied:

$$
\operatorname{det} P=\Phi\left(G, f, k_{y}\right)=0
$$

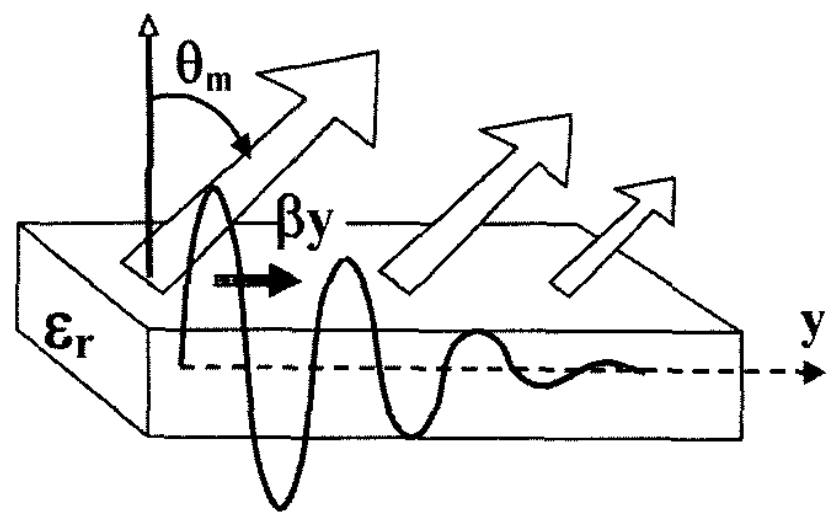

Figure 2. A leaky-wave mode propagating and radiating from a dielectric guide.

Using the Input Parameter window (button 1 of Figure 3), the user must also introduce the parameters for the computation of $\Phi$ : the frequency of analysis, $f$, and the range of values, $\varepsilon_{\text {reff }}$, for which the function $\Phi\left(k_{y}\right)$ will be computed in the real $\beta_{y}$ axis. In this way, by pressing the Show Det-P button (number 2 in Figure 3), the function $\Phi\left(k_{y}\right)$ will be computed and the results will be plotted in the window shown at the bottom of Figure 3. A given solution (a mode of the waveguide) of the analyzed waveguide exists each time this graph crosses the zero level. Using the Find Mode button (number 3 in Figure 3), the user can find the chosen zero of $\Phi\left(k_{y}\right)$, which corresponds to a permitted mode. As it will be explained throughout this paper, a search can be conducted for two different types of modes, namely, closed waveguide modes and open waveguide modes. These can be selected using the controls associated with the Find Mode button. For instance, Figure 3 shows that the program has found three modes in a closed microstrip waveguide. Once a given $k_{y}$ solution

has been found, its fields (currents, electric field, magnetic field, power density, radiation pattern) can be plotted in one or two dimensions using the controls associated with the Fields button shown in Figure 3. The field distributions will be plotted in new windows, which can be saved or printed. Besides this, the solution found can be used to make a dispersion analysis, which can be done using the frequency as the sweep parameter, or by sweeping any geometrical parameter of the structure. The button labeled Dispersion in Figure 3 is used for this purpose.

Figure 4 shows the functional block diagram of the program. In the next sections, some examples will illustrate the working principles of this program, and the types of results that can be obtained for teaching the leaky-wave phenomenon and its applications.

\section{Real Modes in Closed Waveguides}

To more easily understand the theory involved in the propagation of modes in open waveguides, it is better to start by studying the corresponding completely shielded waveguide, and later proceed by opening it. To illustrate this, a uniform strip- 


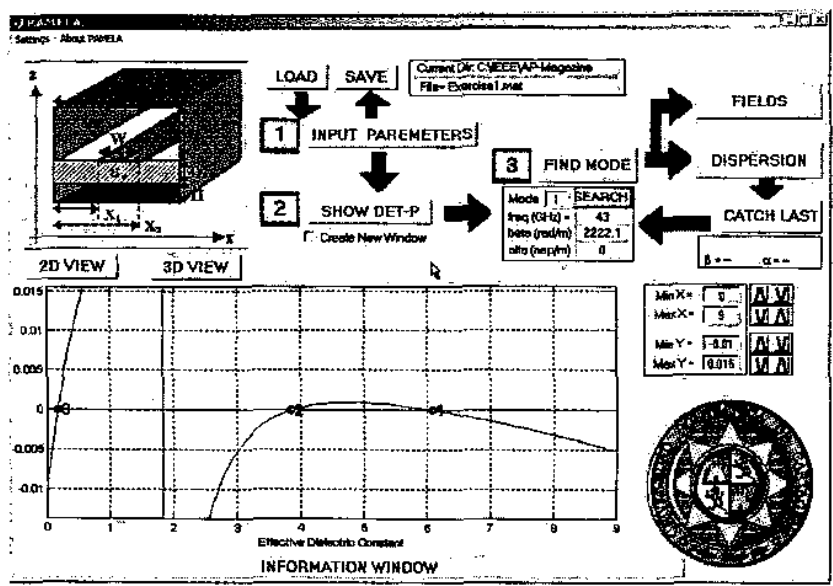

Figure 3. The main window of the $P A M E L A$ graphical user interface.

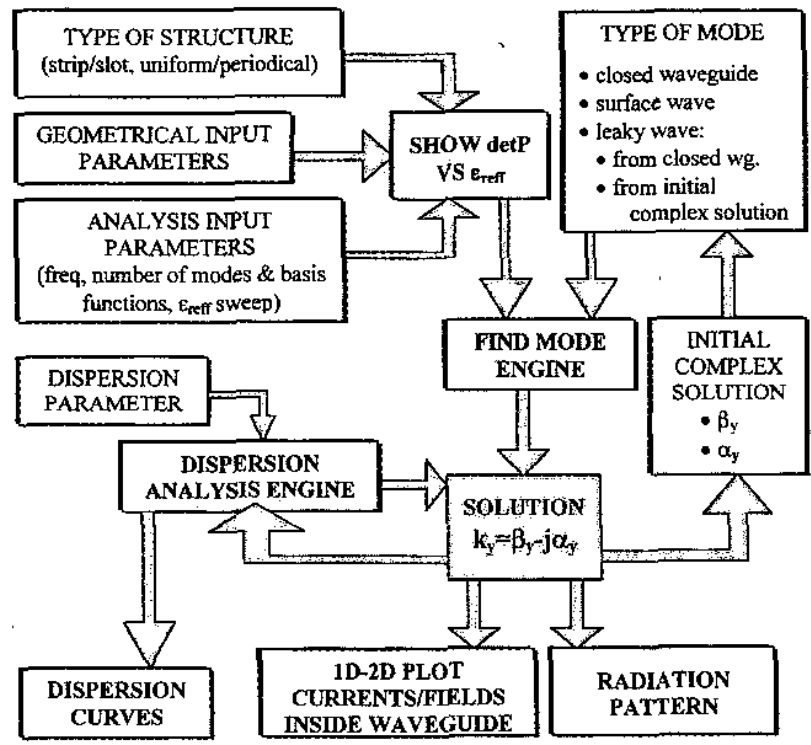

Figure 4. A functional block diagram of $P A M E L A$.

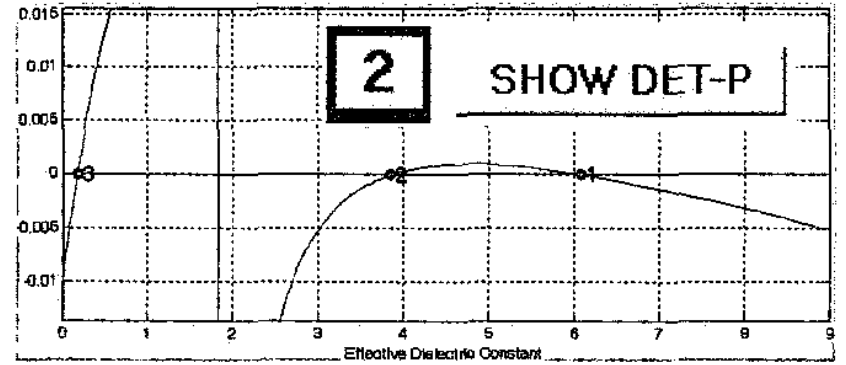

Figure 6. The computation and plot of the determinant of the MoM matrix (det $P$ ) for the closed waveguide loaded with a symmetrically located strip. Three different real modes are observed at a frequency of $43 \mathrm{GHz}$.

IEEE Anternas and Propagation Magazine, Vol. 48, No. 4, August 2006
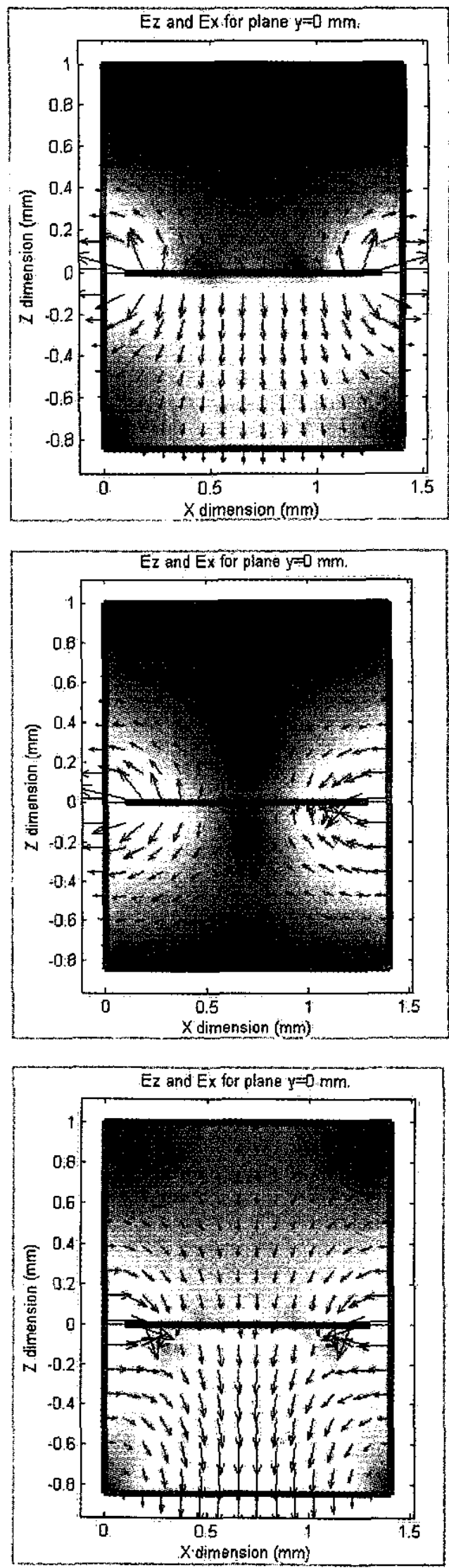

Figure 9. The transverse components of the electric field of the three real modes in the closed structure: (top) Mode 1, quasistatic; (middle) Mode 2, channel guide; (bottom) Mode 3, Perturbed $\mathrm{TE}_{\mathrm{T} 0}$. 
loaded dielectric waveguide (such as that shown in Figure lb) will now be analyzed. The user must choose a strip-type structure and insert the values for each geometrical parameter. This is done using the Input Parameter window shown in Figure 5, which is associated with button 1 of the main window (Figure 3). Figure 5 also shows the dimensions of the proposed structure for this first exercise.

The proposed completely-closed dielectric guide has a relative dielectric permittivity $\varepsilon_{r}=9$, with a strip of width $W=X_{2}-X_{1}=1.2 \mathrm{~mm}$ located symmetrically with respect to the lateral metal walls. The walls are separated by a distance $a=1.4 \mathrm{~mm}\left(X_{1}=0.1 \mathrm{~mm}, X_{2}=1.3 \mathrm{~mm}\right)$. As commented on above, the kemel of the program computes the determinant of a matrix obtained by a numerical procedure (the Method of Moments). This is obtained for a range of values of the effective relative dielectric constant, $\varepsilon_{r e f f}$, related to the longitudinal propagation constant $k_{y}$ by Equation (1). $\varepsilon_{\text {reff }}$ will be swept from $\varepsilon_{\text {reff }}=0$ to $\varepsilon_{\text {reff }}=9$, which is the range of all possible real positive values for $\varepsilon_{r e f f}$, since the dielectric substrate used had $\varepsilon_{r}=9$. The analysis is performed at a fixed frequency of $f=43 \mathrm{GHz}$. The user must press button 2 of the main window in Figure 3, show Det-P, and the program then calculates the function det $P=\Phi\left(k_{y}\right)=\Phi\left(\varepsilon_{\text {reff }}\right)$. It then plots a graph at the bottom of the main window with the real and the imaginary parts of the determinant, as shown in Figure 3. A detail of this graph is presented in Figure 6, where it can also be seen that there are three different solutions at this frequency of $43 \mathrm{GHz}$. Each one corresponds to a zero of the function $\Phi\left(k_{y}\right)$, and therefore to a permitted mode of the closed waveguide. These modes are said to be real modes, since they have a real $k_{y}$ solution.

To find a given mode, the user must press the Find Mode button, which corresponds to Step 3 of the main window in Figure 3. A new dialog window appears, in which the type of mode to be searched must be selected (Figure 7). In this first example, we are interested in the study of real modes, since the structure is completely closed. The user must also choose the order of the mode to be found, the first mode being the dominant mode, that is, the mode that appears at a higher value of $\varepsilon_{\text {reff }}$ in the det $P$ graph.

Figure 7 shows the dialog windows used to find the first mode. Once the user presses the search button of this window, a circle with the order of the mode found appears in the position of the corresponding zero of the $\operatorname{det} P$ graph (see Figure 6). The values of $\beta_{y}$ and $\alpha_{y}$ of the propagation constant of the chosen mode come into view below the Find Mode button, together with the frequency of analysis, as shown in Figure 7.

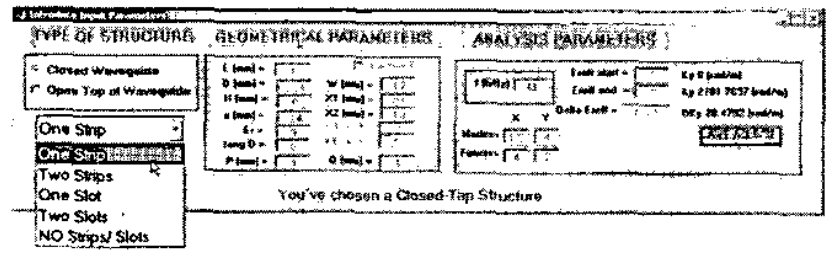

Figure 5. The Input Parameter Window, together with the values of the geometrical and analytical parameters for the first exercise.
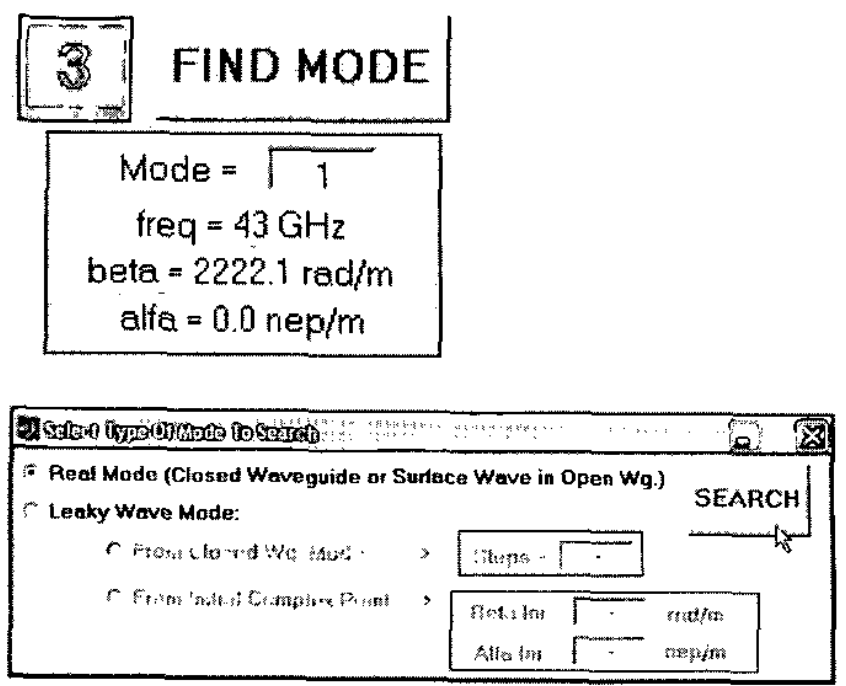

OR

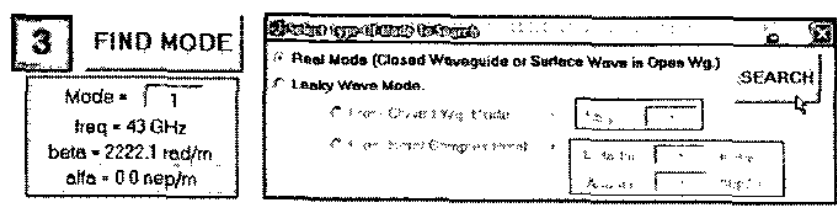

Figure 7. The Find Mode dialog window, showing the values of the first real mode in the closed waveguide.

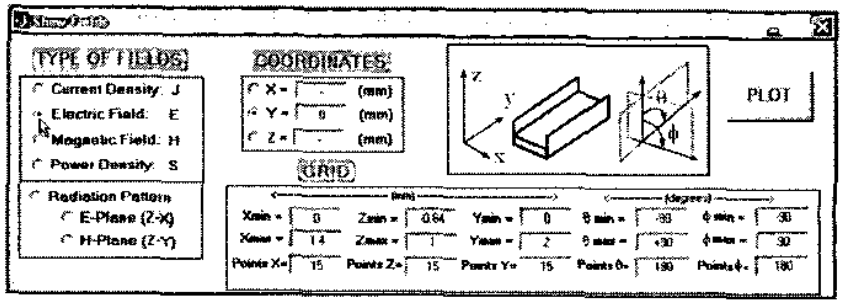

Figure 8. The Show Fields dialog window, showing the controls used to plot the electromagnetic fields of the mode found in the previous step.

Once a given mode has been found, different types of plots of the electric, magnetic, current, and power-density fields can be obtained using the button Fields (Figure 3), which opens the dialog window shown in Figure 8 . This Figure shows how to plot the electric fields in the cross section of the closed waveguide (the $y=0$ plane, transverse $z-x$ coordinates). This window also allows determining the resolution of the grid used to plot the fields.

By pressing the Plot button, new windows appear with the different components of the vector fields. The representation of the fields informs the student about the nature of each mode, and helps the student to understand in a more visual way the structural differences of each type of mode $\{11\}$, as will be illustrated. Figure 9 shows the transverse components ( $x$ - and $z$-directed) of the electric field in the cross section of the waveguide (the plane $y=0$ ) for the three real modes found in the closed strip-loaded structure. 


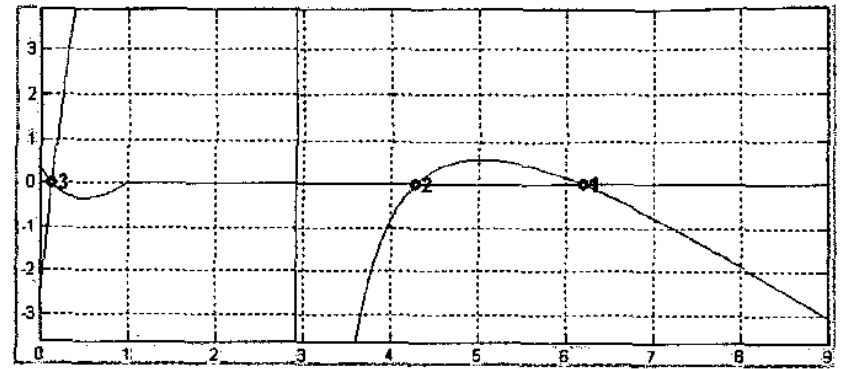

Figure 10. A plot of $\operatorname{det} P$ for the open waveguide loaded with a symmetrically located strip ( $f=43 \mathrm{GHz}$ ).

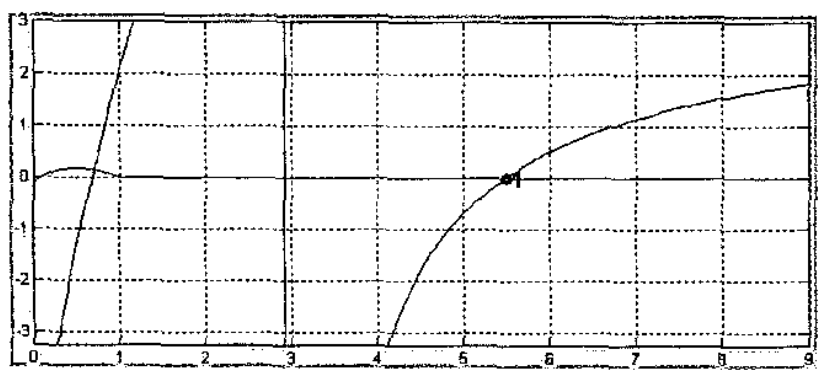

Figure 11. A plot of $\operatorname{det} P$ for the open waveguide loaded with an asymmetrically located strip attached to a side wall $(f=43 \mathbf{G H z})$.

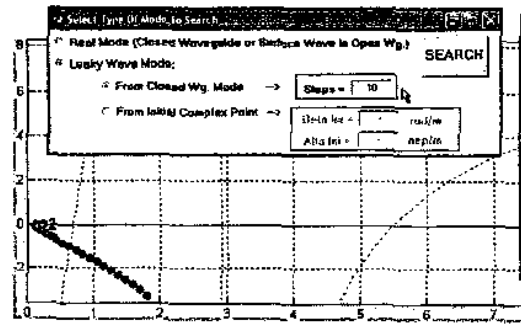

a)

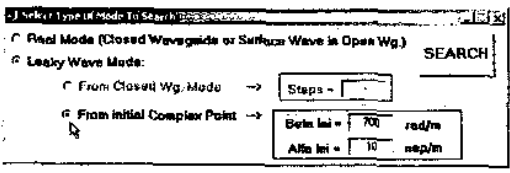

b)

Figure 12. (a) The iterative search for the leaky-wave mode in the asymmetrical open waveguide, starting from the corresponding real mode of the closed waveguide ( $f=43 \mathrm{GHz}$ ) (b) A search using an initial guess of the solution.
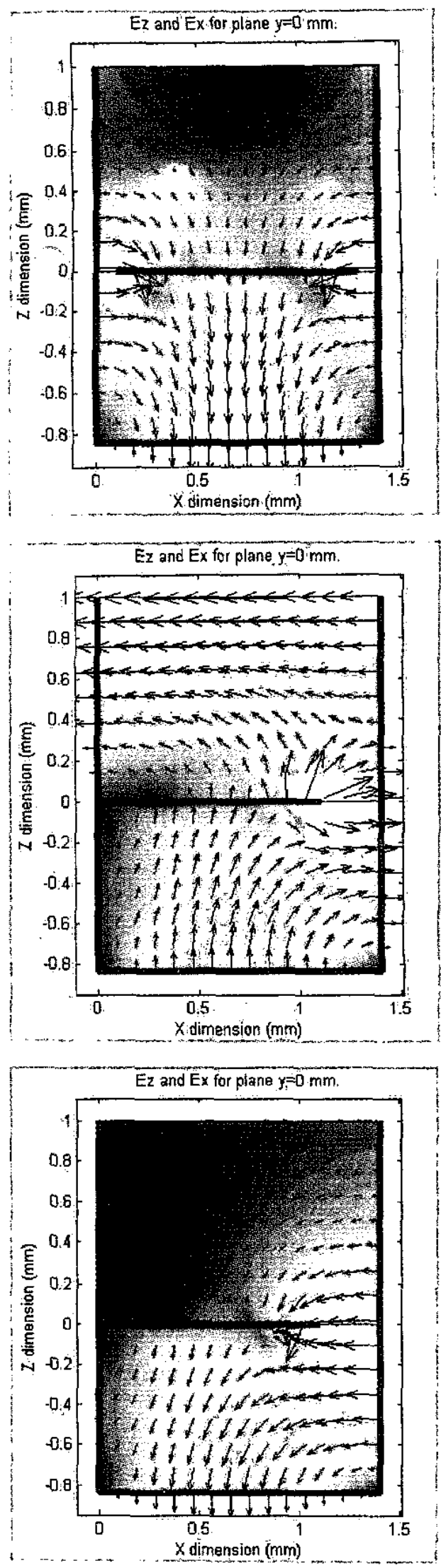

Figure 13. The transverse electric field patterns inside the open waveguide for different scenarios: (a, top) The symmetrically perturbed $\mathrm{TE}_{10}$ mode at $\mathbf{4 3} \mathbf{~ G H z :}$ a surface wave; (b, middle) The asymmetrically perturbed $\mathrm{TE}_{10}$ mode at $43 \mathrm{GHz}$ : a leaky wave; (c, bottom) The asymmetrically perturbed $T E_{10}$ mode at 60 GHz: a surface wave. 
The dominant mode, mode 1, corresponds to the quasi-static solution of the two-conductor structure: that is, a mode the electric field of which is directed from the strip conductor to the rectangular shield walls (if the shield was less close to the strip, and the dielectric thickness, $D$, was lowered, this mode would be the wellknown main mode of a microstrip line.) The second mode is called a channel-guide mode [14], since the electric-field lines emerge from one of the lateral metallic walls and go to the opposite side, as if they were guided between the channel waveguide formed by the two parallel plates. However, this horizontal electric field is perturbed by the strip, which makes the field lines couple to it, as can be seen in Figure 9. The third and last mode is basically the $\mathrm{TE}_{10}$ mode of the rectangular dielectric guide, but perturbed by the two slots created at both sides of the centered strip. It can be seen from Figure 9 that the net electric-field polarization is vertical, in opposition to the net horizontal field of the channel-guide mode. It is important to notice this difference in order to understand their utility for leaky-wave antennas.

\section{Open Waveguides: Leaky-Wave Modes and Surface-Wave Modes}

The strip-loaded dielectric guide is opened on its top side by selecting "Open Top of Waveguide" in the Input. Parameter window (Figure 5). The user must plot the new $\Phi\left(\varepsilon_{\text {reff }}\right)$ function using the show Det-p button, as previously done, obtaining the result shown in Figure 10.

The graph shown seems to be essentially similar to that obtained in Figure 6, observing again three different possible modes in the structure. It looks as if the open waveguide behaves the same as the closed waveguide. However, the student must realize that something very important has happened to $\operatorname{det} P$ for values of $\varepsilon_{\text {reff }}$ below $\varepsilon_{r e f f}=1$. The real part of $\operatorname{det} P$, which was equal to zero for all the range of variation of $\varepsilon_{\text {reff }}$ in the closedwaveguide case (see Figure 6), now differs from zero below $\varepsilon_{\text {reff }}=1$. However, the third zero of $\operatorname{det} P$ goes away, because both the real and the imaginary part of $\operatorname{det} P$ become zero at a given $\varepsilon_{\text {reff }}$ solution below $\varepsilon_{\text {reff }}=1$, producing mode number 3 .

To understand the radiation mechanism, the user must change the position of the strip, which now must be located asymmetrically with respect to the lateral walls and attached to one of the side walls ( $\left.X_{1}=0 \mathrm{~mm}, X_{2}=1.1 \mathrm{~mm}\right)$. The new graph of $\operatorname{det} P$ for this asymmetrically loaded open waveguide is shown in Figure 11.

Two main differences with respect to the symmetrically located strip are observed in the new plot (Figure 10). First of all, it can be seen that there is only one mode above $\varepsilon_{\text {reff }}=1$, which corresponds to the channel-guide mode of the closed structure (Mode 2 in Figures 6, 9, and 10). The fundamental mode of the closed structure (the quasistatic mode) has disappeared, due to the fact that now there is only one conductor in the waveguide (the strip is short-circuited to the side wall). Therefore, the quasistatic solution (which is originated by a potential difference between two different conductors) cannot exist. Second, the third mode of Figures 6 and 10 (the perturbed $\mathrm{TE}_{10}$ mode in Figure 9), which was below the $\varepsilon_{\text {reff }}=1$ limit, has also disappeared. In the asymmetric structure, the real and imaginary parts of det $P$ are both different from zero for $\varepsilon_{r e f f}<1$. This last condition is of great importance, since the solution might not anymore be along the real $k_{y}$ axis, but it can migrate to the complex $k_{y}$ plane. To perform a search of a given complex mode, the user must select the Leaky-Wave Mode search option in the Find Mode window, as shown in Figure 12. This search can be done in two different ways. The user can introduce the coordinates of a given initial point $\left(\beta_{y}\right.$ and $\left.\alpha_{y}\right)$ in the complex plane - which must be an initial guess - from which the program will search for a zero of $\operatorname{det} P\left(k_{y}\right)$. Should the initial guess be close to the final solution of the open waveguide, it is more probable that the program will succeed in the search for the complex solution. Another way consists of the use of the real solution of the closed waveguide as the initial guess to search for the complex mode of the open waveguide. An original iterative algorithm has been implemented [15], in which the top boundary conditions are changed from that of a metal wall (closed waveguide) to that of an open waveguide. If this change is made in small steps, the program can track the variation of the solution from the real $\beta_{y}$ axis to the complex $k_{y}=\beta_{y}-j \alpha_{y}$ plane. For this purpose, the user must introduce the order of the real mode of the closed waveguide to be used as an initial point (see the Mode input box under button 3 in Figure 3), and the number of steps in which the aforementioned iterative search must be executed (see the steps input box in Figure 12).

Figure 12a shows the results obtained for the search of the complex mode by starting from the second mode of the closed asymmetric waveguide. Remember that the quasistatic mode has disappeared, due to the connection of the strip to a lateral wall of the box. In this case, the first mode is the channel-guide mode, and the perturbed $\mathrm{TE}_{10}$ mode being studied becomes the second mode. A new window is opened, showing the step-by-step movement in the complex $k_{y}$ plane, so the student can see how the real mode moves into the complex $k_{y}$ plane as the top wall is opened. The final value of this leaky-wave mode complex propagation constant at $43 \mathrm{GHz}$ is $k_{y}=747.24-j 12.85\left[\mathrm{~m}^{-1}\right]$. Also, this movement is added in the Show Det $\mathrm{P}$ graph of the main window.

The user can also introduce an initial guess for the complex $k_{y}$ solution to be used as a starting point in the search algorithm, as shown in Figure $12 \mathrm{~b}$ (where the point $k_{y}=700-j 10$ was used as initial point). The questions that must be asked at this point are these: Why is the chamel-guide mode a real mode, independently of the waveguide top cover being closed or open? Also, why has the perturbed $\mathrm{TE}_{10}$ mode moved to the complex plane in the case of the asymmetrical open structure, while it was a real mode for the case of the centered-strip open waveguide? A little bit of mathematics is needed to answer these questions. The $z$-directed propagation constant in the vacuum medium for a plane wave can be written using

$$
k_{z}=\sqrt{k_{0}^{2}-k_{x}^{2}-k_{y}^{2}}
$$

with $k_{y}$ being the longitudinal propagation constant of a given mode in the open waveguide studied. Any of the studied modes is determined by a $k_{y}$ value. However, the field variations in the transverse direction cannot be modeled by a simple plane-wave variation. The variation of the field of any mode in the transverse direction is more complicated. Nevertheless, any mode of the laterally-shielded waveguide can be expressed as a contribution of the 
orthogonal set of parallel-plate modes (PPM) supported by the lateral walls (separated at a distance $a$ ). In this way, it can be said that each mode of the open waveguide is created by the convergent summation of many parallel-plate modes, each one characterized by its harmonic variation in the $x$ direction:

$$
k_{x m}=m \frac{\pi}{a}
$$

In the case where the main parallel-plate mode $\left(m=0 \rightarrow k_{x m}=0\right)$ is excited in a given waveguide mode, Equation ( 5 ) can be used to determine its $z$ propagation constant in the vacuum, obtaining the following result:

$$
k_{z, m=0}=\sqrt{k_{0}^{2}-k_{y}^{2}}
$$

This transverse propagation constant must be real in order to allow this main parallel-plate mode to radiate: that is, to be above cutoff in the vacuum medium. Therefore, the radiation condition for the main parallel-plate mode can be written as follows:

$$
\text { Radiation Region } \rightarrow k_{0}>k_{y} \rightarrow \frac{k_{y}}{k_{0}}=\sqrt{\varepsilon_{\text {reff }}}<1 .
$$

Therefore, it can be said that $\varepsilon_{r e f f}=1$ is the limit of the radiation region for the main parallel-plate mode. For higher-order parallelplate modes, this condition is more restrictive: $k_{y}$ must have a still tower value (which can be easily obtained by introducing Equation (6) into Equation (5)). Since any mode of the laterally-shielded open waveguide can be expressed as a summation of parallel-plate modes, it is necessary that at least one of these parallel-plate modes radiate in order to say that the waveguide is radiating. For this reason, it can be said that above $\varepsilon_{\text {reff }}=1 \quad\left(k_{y} / k_{0}=1\right)$, none of the modes of the open waveguide can radiate, since all the energy is below cutoff in the vacuum. In this case, it is said that the mode is a surface-wave mode, having a real $k_{y}$ (and $\varepsilon_{\text {reff }}$ ), which means that all its propagating energy is confined in the dielectric slab. This is the case for the channel-guide mode shown in Figure 11, the $\varepsilon_{\text {reff }}$ of which is real and above unity. The radiation region $\left(k_{y} / k_{0}<1\right)$ is also called the "fast-wave" region, since the mode has a longitudinal phase velocity greater than the velocity of light in vacuum [16].

However, the second question is not yet answered. Figure 10 showed a real solution $\left(\mathrm{TE}_{10}\right.$ mode) below the $\varepsilon_{r e f f}=1$ limit for the symmetrically located strip, while for the asymmetric case (Figure 11), this mode became a complex mode. To understand this phenomenon, the student must obtain the electric-field pattem inside the waveguide for these two cases, shown in Figures 13a and $13 b$.

For the case of the symmetrically perturbed $\mathrm{TE}_{10}$ mode, the main parallel-plate mode $(m=0)$ is not excited, which therefore does not lead to a net radiation. As can be seen from Figure 13a, all the energy is confined in the dielectric-guide region (the bottom of the guide), while in the top, air-filled region, the field is evanescent. On the contrary, for the asymmetrically located strip, the main parallel-plate mode $(m=0)$ is excited and radiation occurs (remember that the $m=0$ case can propagate in the vacuum region). This main parallel-plate mode has no variation in the $x$ direction $\left(k_{x m}=0\right)$, which means that the electric field has a horizontal transverse electric field, going from one of the side walls to the other with a constant amplitude. This constant horizontal-field component can only be excited by the $\mathrm{TE}_{10}$ mode if the perturbation is located asymmetrically, inducing a difference of potential between the two side walls. All these phenomena are clearly shown in Figure 13a and Figure 13b.

At this point, the student should have acquired the following conclusions, relating the change from the modal spectrum of the closed symmetrical waveguide to that of the open asymmetrical waveguide:

1. The attachment of the strip to the side of the waveguide makes the quasistatic mode disappear from the modal spectrum of the waveguide (closed or open).

2. The radiation region of any mode is the so-called "fastwave" region, in which the $\varepsilon_{\text {reff }}$ value is below unity.

3. To make the mode radiate (a leaky-wave mode), the main parallel-plate mode $\left(k_{x, m=0}=0\right)$ of the laterally shielded waveguide must be excited, since this mode is the only mode above cutoff in the air region, and therefore responsible for the radiation mechanism.

4. The $\mathrm{TE}_{10}$ mode of the rectangular dielectric guide must be perturbed asymmetrically to make it radiate, since the asymmetry is responsible for the excitation of the main parallel-plate mode $(m=0)$. If the planar perturbation (strip or slot) is located symmetrically with respect to the side walls, the mode is a surface-wave mode, even in its "fast-wave" region, since the main parallel-plate mode $(m=0)$ is not excited.

\section{Frequency Dispersion: Radiation From Leaky-Wave Modes}

All previous results have been obtained for a fixed frequency value $(43 \mathrm{GHz})$. The frequency dispersion of the leaky-wave modes must be studied in order to understand the behavior of the open waveguide for different frequencies. Once a given mode has been found at a fixed frequency, a dispersion analysis can be performed using the PAMELA environment. The user must press the Dispersion button of the main window, and a new Dispersion Analysis window (see Figure 14) will open. The user must select which parameter to sweep (in our case, it is the frequency), the range of variation (in our case, from $43 \mathrm{GHz}$ to $100 \mathrm{GHz}$ ) and the type of Figures to plot ( $k_{y}$ in the complex plane, $\beta_{y}, \alpha_{y}$, and/or radiation angles: the beam direction, $\theta_{m}$, and the beamwidth, $\Delta \theta$ ). By pressing the Compute button, the program will start to make the simulations and will plot the results.

Figure 14 shows the results window obtained in order to see the variation of the $k_{y}$ solution in the complex plane for the perturbed $\mathrm{TE}_{10}$ leaky-wave mode as the frequency is varied from $10 \mathrm{GHz}$ to $100 \mathrm{GHz}$. It can be seen that the solution becomes real ( $k_{y}=\beta_{y}, \quad \alpha_{y}=0$ ) for frequencies above $44.5 \mathrm{GHz}$. This phenomenon is called leaky-to-surface-wave transition, and occurs 


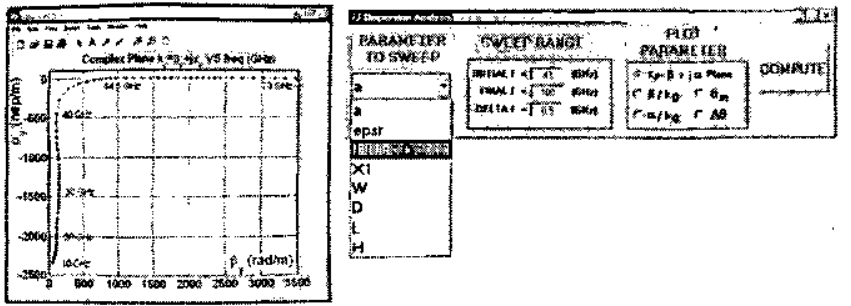

OR
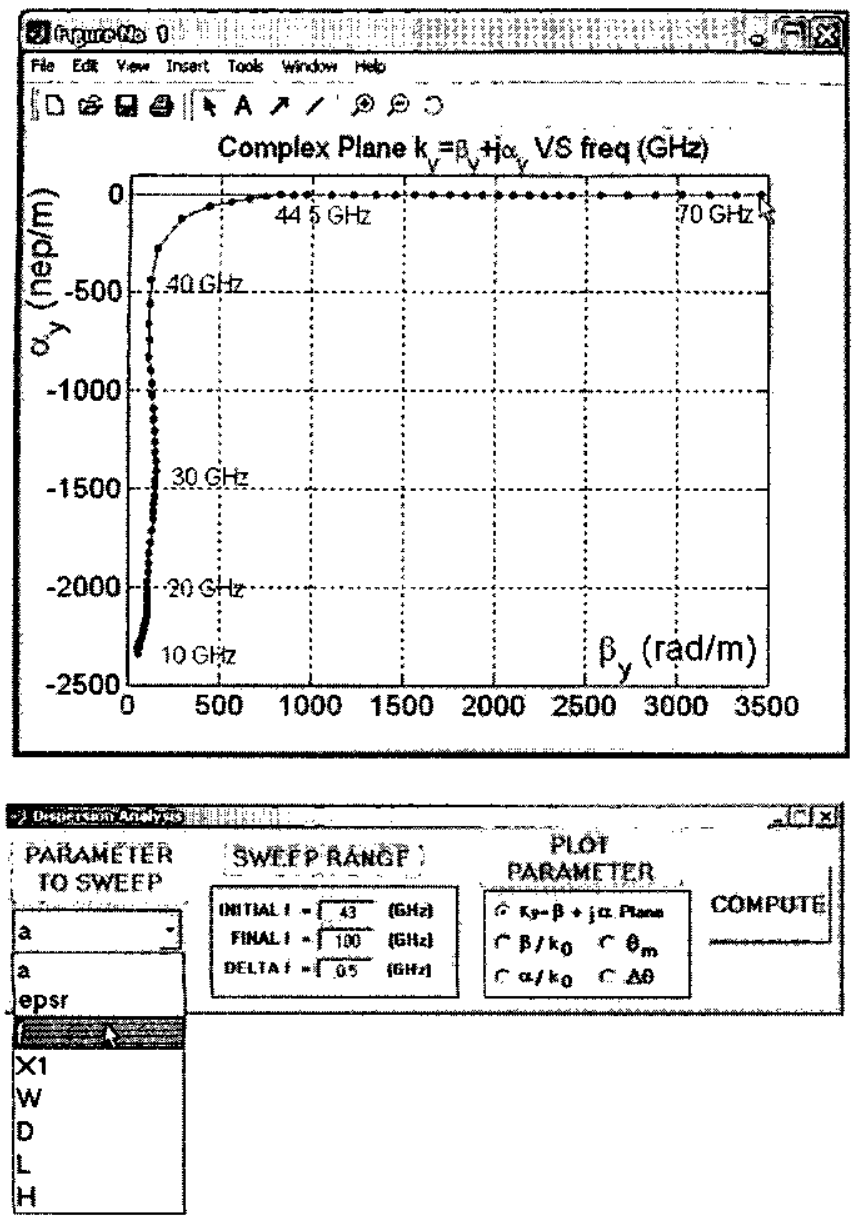

Figure 14. The Dispersion Analysis window, used to perform a dispersion analysis with frequency in a sweep range from $43 \mathrm{GHz}$ to $100 \mathrm{GHz}$. The variation of the $k_{y}$ solution in

the complex $k_{y}$ plane is obtained in a new window, shown containing the plot.

in all open dielectric waveguides [16]. By observing separately the evolution of the real and the imaginary parts of $k_{y}$, and expressing them normalized with respect to $k_{0}$, new and interesting conclusions can be obtained.

Figure 15 a shows the dispersion with frequency $(0-100 \mathrm{GHz})$ of the normalized phase constant for the perturbed $\mathrm{TE}_{10}$ leaky mode $\left(\beta_{y} / k_{0}\right)$. The student is asked to compute the well-known frequency dispersion of the $T E_{10}$ mode of the completely closed rectangular dielectric guide used in this structure (width $a=1.4 \mathrm{~mm}$, height $D=0.84 \mathrm{~mm}$, substrate $\varepsilon_{r}=9$ ), and to plot it together with the perturbed $\mathrm{TE}_{10}$ mode obtained with the program. The $\mathrm{TE}_{10}$ mode curve is plotted in Figure 15a with a dashed line, while the perturbed $\mathrm{TE}_{10}$ mode is represented with a continuous line. In this way, the student can see how both dispersion curves behave in a similar way, the perturbed mode having a lower phase constant due to the fact that the field propagates in an inhomogeneous medium, between the dielectric slab and the air region. However, an important difference is seen in Figure 15a. When the real $\mathrm{TE}_{10}$ mode reaches its cutoff zone (below $35 \mathrm{GHz}$ ) the phase constant becomes zero, since the propagation constant becomes purely real. However, for a leaky-wave mode, the normalized phase constant reaches a stable value different from zero below a given frequency, which seems to be related to the cutoff region.
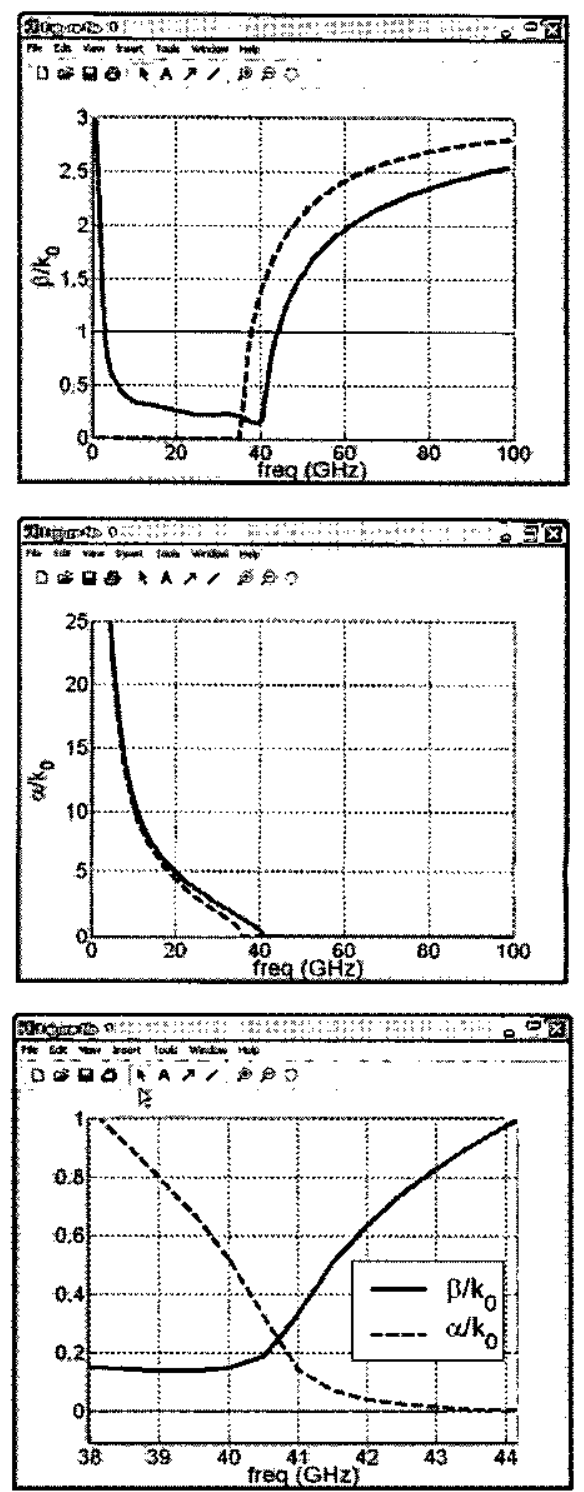

Figure 15. The dispersion of the normalized phase and attenuation constants with frequency: (a, top) the normalized phase constant for the perturbed $\mathrm{TE}_{10}$ leaky mode; (b, middle) the attenuation constant for both the analytical $\mathrm{TE}_{10}$ mode of the dielectric rectangular closed waveguide (dashed line) and the perturbed leaky-wave $\mathrm{TE}_{10}$ mode of the open waveguide (continuous line); (c, bottom) a detailed plot of $\beta / k_{0}$ and $\alpha / k_{0}$ for the perturbed $\mathrm{TE}_{10}$ leaky-wave mode. 

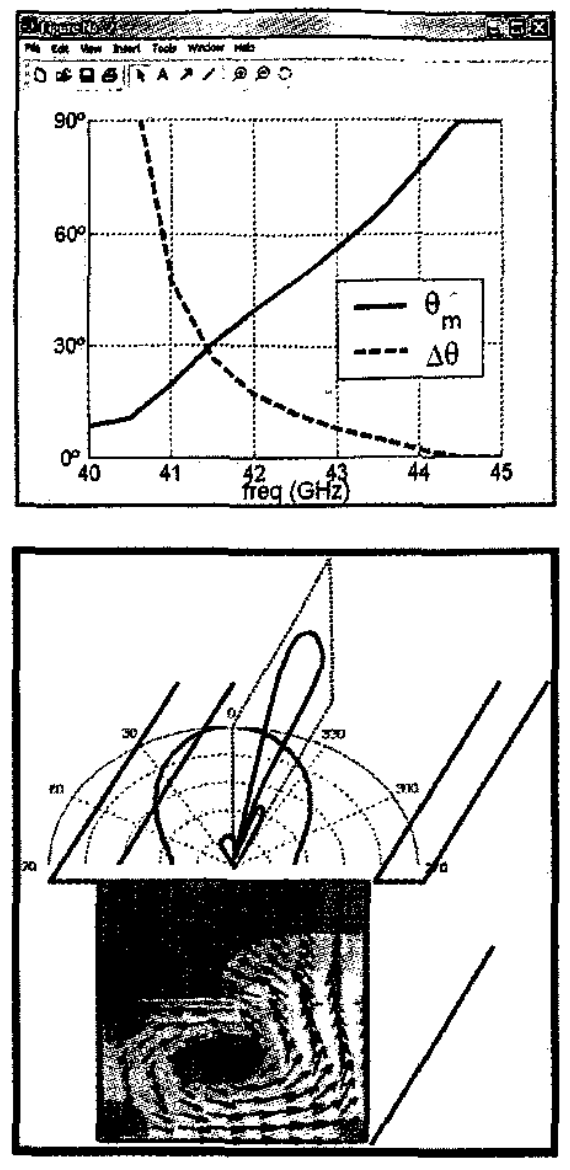

Figure 16. (a, top) The variation of the main direction of the beam and the beamwidth with frequency. (b, bottom) The power-density pattern inside the waveguide and radiated by the perturbed $\mathrm{TE}_{10}$ leaky mode $(f=43 \mathrm{GHz})$.

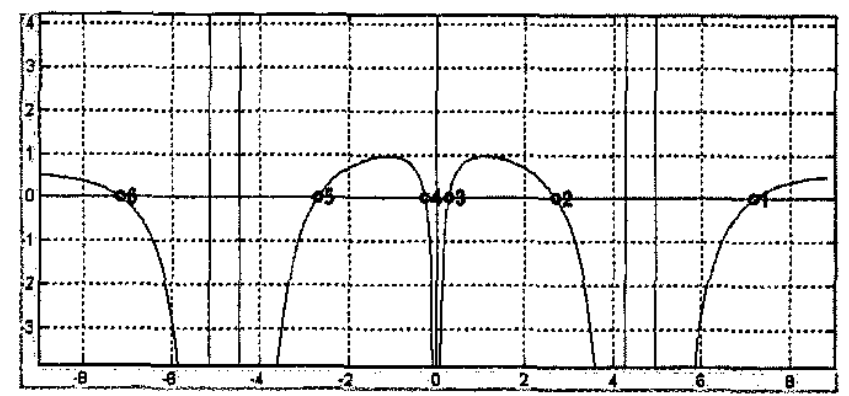

Figure 18. A plot of $\operatorname{det} P$ for the closed periodically striploaded dielectric guide $(42 \mathrm{GHz})$.

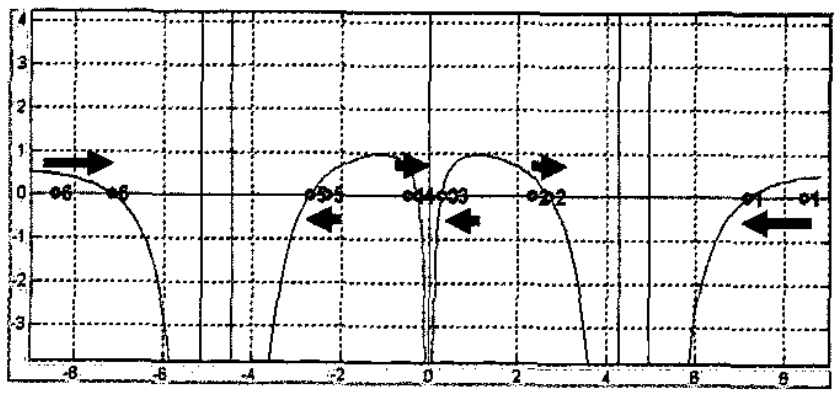

Figure 19. The evolution with frequency of the zeros for the periodic closed guide, from $40 \mathrm{GHz}$ to $42 \mathrm{GHz}$. 
Figure 15b shows the variation of the attenuation constant for both the analytical $\mathrm{TE}_{10}$ mode of the dielectric rectangular closed guide (the dashed line), and the perturbed leaky-wave $\mathrm{TE}_{10}$ mode of the open waveguide (the continuous line). Again, these two plots are quite similar, a rapid increase of $\alpha / k_{0}$ being observed as the frequency decreases in the cutoff region. However, the cutoff region and the radiation region of a leaky-wave mode must not be confused. For this purpose, Figure $15 \mathrm{c}$ shows a detailed plot of $\beta / k_{0}$ and $\alpha / k_{0}$ for the perturbed $\mathrm{TE}_{10}$ leaky-wave mode. Above $44.5 \mathrm{GHz}, \alpha_{y} / k_{0}$ becomes zero, which is due to the conversion of the mode to a surface wave, therefore eliminating the radiation phenomenon. Below this transition frequency, $\alpha_{y} / k_{0}$ rises in a smooth way. This region is the radiation region, the upper limit of which was established by Equation (8). Moreover, below $41 \mathrm{GHz}$, the attenuation constant increases in a rapid way, following the typical curve of the imaginary part of a $\mathrm{TE}_{10}$ mode below cutoff in a standard waveguide (see Figure 15b). The attenuation constant, $\alpha_{y}$, can be divided into two different parts, namely the reactive attenuation constant and the radiative attenuation constant:

$$
\alpha_{y}=\alpha_{y}^{R A D}+\alpha_{y}^{R E A C T}
$$

Both parts contribute to the decreasing-amplitude behavior of the mode as it propagates along the waveguide axis. However, the reactive attenuation corresponds to the mode carrying reactive power (the mode below cutoff), while the radiative attenuation part accounts for the radiation losses (the energy radiated into space). In the cutoff region, $\alpha^{R E A C T}$ dominates the rate of radiation losses. This region can also be limited by the next condition, which has been checked by numerical measurements [17]:

$$
\text { Cutoff Region } \rightarrow \frac{\alpha_{y}}{k_{0}}>\frac{\beta_{y}}{k_{0}}
$$

This last condition, together with the radiation upper limit (the transition to surface wave), allows gathering the limits of the radiation region of a leaky-wave mode in a numerical expression, once the values of the phase and attenuation constants are known:

$$
\begin{aligned}
& \text { Radiation Region Limits of a Leaky-Wave mode } \\
& \rightarrow \frac{\alpha_{y}}{k_{0}}<\frac{\beta_{y}}{k_{0}}<1
\end{aligned}
$$

These limits establish the practical frequency bandwidth of radiation of a leaky-wave antenna. Figure $13 \mathrm{c}$ shows the electric-field pattern for the perturbed $\mathrm{TE}_{10}$ mode at $60 \mathrm{GHz}$. At this frequency, the mode has made the transition to a surface wave: it can be seen from the plot that the energy is confined in the dielectric region, and no fields propagate through the vacuum region. The spectral gap associated with the transition between the leaky and surface regimes [16] can also be found by PAMELA. In this region, a mode can be leaky even when it is in the slow-wave zone. However, this regime is out of the scope of the illustrative examples described in this paper. The radiation pattern of a leaky-wave mode is that of an exponentially decreasing illuminated aperture, resulting in a narrow beam radiating at a given angle $\theta_{m}$, measured from the broadside direction (see Figure 2). The resulting main-beam direction $\left(\theta_{m}\right)$ and the $3 \mathrm{~dB}$ beamwidth $(\Delta \theta)$ can be approximated by [6]

$$
\begin{aligned}
& \sin \theta_{m} \cong \frac{\beta_{y}}{k_{0}}, \\
& \Delta \theta \cong \frac{1}{\frac{L_{A}}{\lambda_{0}} \cos \theta_{m}} \approx \frac{\alpha_{y} / k_{0}}{0.183 \cos \theta_{m}} .
\end{aligned}
$$

These expressions are used by the program to compute the characteristics of the main radiated beam from the phase and attenuation constants of a leaky-wave mode. Figure 16 shows the results obtained from the perturbed $\mathrm{TE}_{10}$ mode in its radiation bandwidth (41-44.5 GHz). A plot of the transverse power density flux inside the waveguide and the numerical radiated patterns ( $E$ and $H$ planes) are also shown in Figure 16. It can be seen that the energy flows from the dielectric guide to the air region. The parallel-plate stub is needed to focus the energy, avoiding unwanted grating lobes and cross polarization. How the field emerging from the dielectric guide has a reactive zone, above which the electric-field polarization becomes purely horizontal, can bc seen from Figure $13 \mathrm{~b}$. Figure $16 \mathrm{~b}$ it is also seen that the outgoing power flux lines are focused by the parallel-plate stub once they have propagated to a given height. The computed radiated pattem, observed in Figure $16 \mathrm{~b}$, is that of a narrow beam, tilted at an elevation angle $\theta_{m}$ in the $\mathrm{H}$ plane (the $y-z$ plane), with some secondary lobes. This narrow beam in the $\mathrm{H}$ plane is due to the highly effective radiating surface of a leaky-wave antenna, obtained from the exponentially decaying illumination of the waveguide aperture. From Equation (13), it is seen that as the attenuation rate, $\alpha_{y}$, is lower, the radiated beam is narrower, obtaining higher antenna directivities. In the $\mathrm{E}$ plane (the $x-z$ plane), a not-so-narrow beam, directed broadside with respect to the azimuth angle, $\phi$, is obtained. Array techniques can be used to increase the directivity in this $\mathrm{E}$ plane.

Two further important conclusions about leaky-wave antenna can be obtained from Figure 16a. According to Equation (12), the angle of maximum radiation, $\theta_{m}$, depends directly on the normalized phase constant, $\beta_{y} / k_{0}$, of the leaky mode. Since the phase constant of a leaky-wave mode (in our case, the perturbed $T E_{10}$ mode) is clearly dispersive as a function of the frequency, the pointing direction of a leaky-wave antenna is scanned with the frequency. In particular, and according to Equation (8) and the variation of $\beta_{y}$ observed in Figure 15c, it can be seen in Figure 16a how this $\theta_{m}$ angle is scanned from near the broadside direction, obtained at the lower-frequency limit $\left(\theta_{m}=12^{\circ}\right.$ at $40.5 \mathrm{GHz}$ ), to the end-fire direction, which is reached near the transition to a surface wave $\left(\theta_{m}=90^{\circ}\right.$ at $\left.44.5 \mathrm{GHz}\right)$. This is the first important feature of a leaky-wave mode: the frequency-scanning-angle behavior. This can be of much interest in some applications (e.g., radar and tracking), but it can also limit the bandwidth for fixed-beam applications. The other interesting characteristic is related to the beamwidth frequency response. In Figure 16a, it is seen that the beamwidth, $\Delta \theta$, strongly varies with the frequency, having a narrower beam as the frequency increases. In the cutoff region (below $41 \mathrm{GHz}$ ), the beamwidth seems to rise due to the rapid increase of $\alpha_{y}$, due to the reactive attenuation. However, the radiated beamwidth formula has no physical meaning outside the radiation region. The variation of the beamwidth with the frequency can be an annoying effect in many applications where the same directivity is needed for different pointing directions. 
At the end of this second exercise, the student should have understood the following important topics relating to the behavior of leaky-wave modes:

1. Leaky-wave modes in open dielectric waveguides have an effective radiation-frequency bandwidth, limited by the cutoff region and the surface-wave region. The limits of these regions can be obtained from the leaky-wave mode phase and attenuation constants.

2. The attenuation constant of a leaky-wave mode can be divided into two different contributions, one due to reactive attenuation and the other due to radiative losses, the first being dominant in the cutoff region.

3. Leaky-wave antennas have large bandwidths, due to their traveling-wave nature. This is in contrast to the resonance-radiation mechanism of other types of antennas (printed-circuit patches or slots), the bandwidths of which are much more limited.

4. High gains in the $\mathrm{H}$ plane can be obtained from a leakywave antenna by exciting a weakly attenuated leaky. wave mode. However, arrays of leaky-wave antennas must be used to enhance the low directivity in the $\mathrm{E}$ plane.

5. Dielectric-filled leaky-wave antennas have the capability to scan the pointing direction with frequency, from near-broadside to end-fire. The beamwidth also varies with the frequency.

\section{Uniform Leaky-Wave Antenna Design}

Once the student is familiar with the leaky-wave phenomenon in open dielectric waveguides, we can start with the design of a uniform leaky-wave antenna. The main idea for a versatile leakywave antenna design is to find a structure in which the leakage rate $\left(\alpha_{y}\right)$ of a given leaky-wave mode can be varied while maintaining unchanged its phase constant $\left(\beta_{y}\right)$. This is necessary for three practical reasons:

1. According to Equations (12)-(13), if one can change $\alpha_{y}$ without perturbing $\beta_{y}$, the beanwidth can be controlled in a wide range (from narrow to wide beams), independently of the scan angle of the antenna.

2. Moreover, the illumination of a practical leaky-wave antenna must be adjusted to a given pattern in order to control sidelobes [6]. For this purpose, the attenuation constant, $\alpha_{y}$, is varied along the length of the antenna.

However, the antenna must radiate at the same angle at any point, which means that $\beta_{y}$ must remain constant all along the antenna's length for the different values of $\alpha y$

3. When feeding a leaky-wave antenna, the source must inject power into the open waveguide. To avoid direct radiation from the source discontinuity and to obtain an optimum matching, it is desirable that the input section of the antenna does not radiate. From this non-radiative waveguide $\left(\alpha_{y}=0\right)$, the section of the leaky-wave antenna must be smoothly varied to obtain the final radiation rate $\left(\alpha_{y} \neq 0\right)$. In this variation of $\alpha_{y}$, the phase constant of the leaky-wave mode must remain unchanged in order to radiate at a single angle, avoiding mismatch between the source and the antenna.

Not all the leaky-wave modes allow their phase and attenuation constants to be controlled in such an independent fashion. Moreover, the leaky-wave antenna designer must find which geometrical parameter of the cross section of the antenna can be modified to vary $\alpha_{y}$ in a wide range while not perturbing $\beta_{y}$. Besides this, the parameter chosen to control the radiation of the antenna must be modulated along the length of the antenna for a practical tapered design. It is of much practical interest that the modification of this parameter of the open waveguide be mechanically simple to realize. Therefore, the design of a practical and versatile leakywave antenna is not a trivial task, and many research works have been published on this topic. Using the PAMELA tool, a recent and original type of hybrid planar-waveguide technology leaky-wave antenna design has been proposed [18]

With this introduction, the student is asked to use the Dispersion Analysis module of $P A M E L A$ to vary different parameters of the structure (the waveguide width, $a$; the height, $D$; the dielectric-substrate permittivity, $\varepsilon_{r}$; the strip position, $X_{1}$, and width, $W$; etc.), trying to control the leakage constant of the leakywave mode without affecting its phase constant. The first question is how to choose which leaky-wave mode is best suited to be used as the radiation mechanism of a practical leaky-wave antenna. In the strip-loaded dielectric leaky-wave antenna, three different
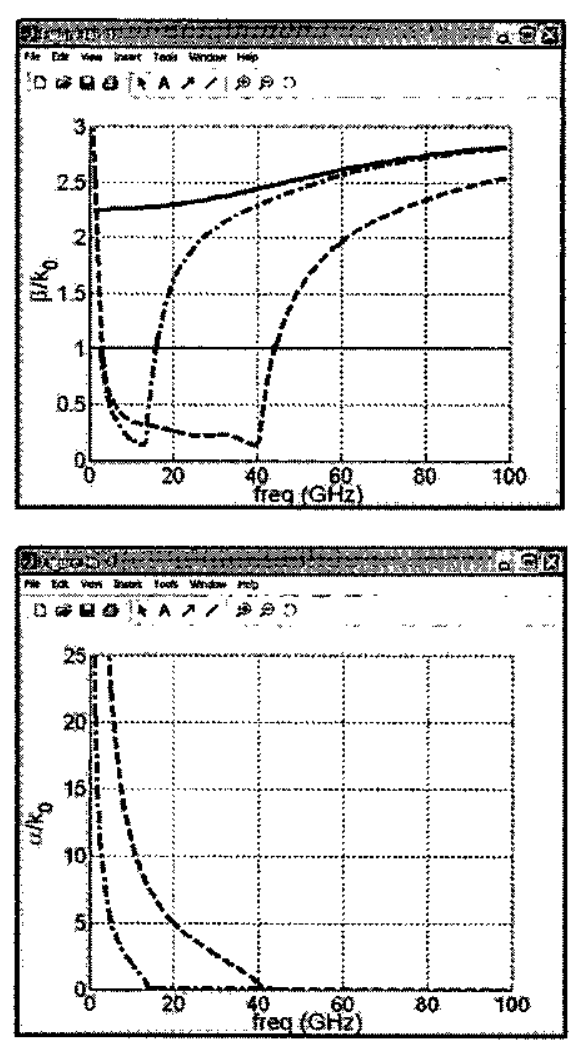

Figure 17. The frequency dispersion of the propagation constants of the different modes in the strip-loaded leaky-wave antenna. 
modes were found (see Figure 9), namely, the quasistatic mode, the channel mode, and the perturbed $\mathrm{TE}_{10}$ mode, in order of appear-ance. Their propagation-constant frequency dispersions are plotted in Figure 17.

The quasi-static mode is the main mode of the open wavegutde. It can be seen from Figure 17 (the continuous line) that this mode's normalized phase constant, $\beta_{y} / k_{0}$, is always greater than unity. Therefore, this mode cannot radiate, and must be avoided for uniform antenna applications. For this reason, the strip was connected to the side wall, making this mode disappear from the modal spectrum of the waveguide. The other two modes have a radiation range. The dominant leaky-wave mode is the channelguide mode (the dotted-dashed line), while the second leaky-wave mode to appear is the perturbation of the $\mathrm{TE}_{10}$ mode (the dashed line). Although the two modes radiate in their corresponding radiation band (around $17 \mathrm{GHz}$ and $43 \mathrm{GHz}$, as can be seen in Figure 17 from the range corresponding to Equation (11)), the $\mathrm{TE}_{10}$ mode is more suited to being used in antenna applications than is the channel-guide mode. The reason is that the $\mathrm{TE}_{10}$ mode radiation can be better controlled (by varying $\alpha_{y}$ without changing $\beta_{y}$ ). This is due to the fact that the channel guide excites the main parallel-plate mode ( $m=0$ ) independently of the strip position or width, since the net electric field of the channel guide is directed from one side wall to the other (horizontal electric field), as can be seen in Figure 9 for Mode 2 . In contrast, the $\mathrm{TE}_{10}$ mode is vertically polarized, and must be asymmetrically perturbed to excite the main parallel-plate mode $(m=0)$, as was shown in Figures 13a and 13b. In this way, by controlling the strip position or width the leakage rate can be controlled from zero (when the strip is symmetrically located) to high values (for a high degree of asymmetry). This control is not possible for the channel-guide mode. It can therefore be said that the $\mathrm{TE}_{10}$ mode allows controlling the radiation rate by the asymmetry principle, while the channel guide does not.

With this third exercise, the student should have leamed the following concepts:

1. A versatile leaky-wave antenna design is based on radiation from a leaky-wave mode, the phase and leakage constants of which can be controlled independently.

2. For this purpose, the different types of open waveguide must be examined to find a geometrical parameter of its cross section that can perform the aforementioned control of the radiation.

3. Moreover, not all the leaky-wave modes of a given open waveguide can be controlled in the same way. For instance, the perturbed $\mathrm{TE}_{10}$ mode of a dielectric guide is better suited for antenna applications than a channel-guide mode.

\section{Periodic Leaky-Wave Antennas: Excitation of Space Harmonics}

The second basic type of leaky-wave antenna is based on periodic structures, sharing some similarities but also having some differences with respect to a uniform leaky-wave antenna [6]. The $P A M E L A$ environment allows analyzing periodically perturbed dielectric guides, just by introducing different values for the unitcell length (parameter $P$ in Figure 1c) and for the strip or slot length (parameter $Q$ ) in the Input Parameter window (Figure 5). In this example, the parameters of the proposed periodic strip-loaded guide are $P=3.38 \mathrm{~mm}, Q=2.366 \mathrm{~mm}, L=1 \mathrm{~mm}$, $D=1.4 \mathrm{~mm}, \quad H=0 \mathrm{~mm}, \quad \varepsilon_{r}=9 \mathrm{~mm}, \quad W=0.84 \mathrm{~mm}, \quad$ and $f=42 \mathrm{GHz}$, which are those of a practical published design [19]. The student must be introduced to the basic theory of space harmonics before the practical session [20].

When the cross section of a structure is not uniform, but instead it is of periodic nature, it cannot be properly said that the modal solution of such structures is described by the given modal longitudinal wavenumber. However, it can be said that the solutions of a periodic structure can be expressed by an infinite summation of space harmonics, the phase constant of all of them being related by the following expression:

$$
\beta_{y n}=\beta_{y 0}+n \frac{2 \pi}{P}, n=-\infty, . .,-2,-1,0,+1,+2, . .,+\infty,
$$

with $P$ being the period of the structure (the length of the unit cell). Once the phase constant of the main space harmonic $\left(n=0 \rightarrow \beta_{y 0}\right)$ is known, the propagation constant of the set of higher-order space harmonics can be determined by Equation (14). In this way, it can be said that the main space-harmonic wavenumber value is a representative solution for the periodic structure, as is done for the modal solutions of a uniform guide.

The student is asked to study the closed periodic structure by computing the det $P$ function sweeping $\varepsilon_{\text {reff }}$ from -9 to 9 , since the substrate relative permittivity is $\varepsilon_{r}=9$ (we will explain-later the physical meaning of having negative values for $\beta_{y}$ and $\varepsilon_{\text {reff }}$ ). Once the Show Det- $\mathrm{P}$ button has been pressed and all the zeros are searched, the aspect of the main window det $P$ graph obtained is shown in Figure 18.

Six real solutions (six zeros of $\operatorname{det} P$ ) are found at the frequency of $42 \mathrm{GHz}$ and between the proposed limits for $\varepsilon_{\text {reff }}$. However, the student must learn to interpret the physical meaning of these solutions. For this purpose, it is necessary to perform a new analysis at a different but close frequency, and to again find the zeros, in order to observe the frequency behavior of these solutions. Figure 19 shows the same plot as Figure 18, but having added the position of the zeros found at $40 \mathrm{GHz}$. The arrows indicate the evolution of the zeros in the increasing frequency direction, from $40 \mathrm{GHz}$ to $42 \mathrm{GHz}$.

At a first glance, the student could think that six different solutions exist in this periodic structure; however, all of them are related. The zeros found with numbers 2,4 , and 6 are related by Equation (14), and are therefore part of the same set of space harmonics. Mode $2\left(\beta_{y 0}=+1275.2 \mathrm{rad} / \mathrm{m}\right)$ is the main space harmonic, and modes 4 and 6 are the space harmonic of indexes $n=-1$ and $n=-2$, respectively:

$$
\beta_{y n}=1275.2+n \frac{2 \pi}{3.38} 10^{3} \mathrm{rad} / \mathrm{m}
$$

On the other hand, modes 1, 3, and 5 are also related by Equation (14), which determines the space-harmonic order of each one (mode 5 is $n=0$, mode 3 is $n=-1$, and mode 1 is $n=-2$ ): 


$$
\beta_{y n}=-\left(1275.2+n \frac{2 \pi}{3.38} 10^{3}\right) \mathrm{rad} / \mathrm{m}
$$

Moreover, Equations (15) and (16) are related, since one equation is the other equation but with the sign changed. The student must remember that a given modal solution of the inhomogeneous Maxwell's equations has two variants, the progressive and the regressive variants, having the sign changed on their phase constants. The physical meaning of each solution is that of an ingoing or an outgoing wave with respect to the selected waveguide direction (the $y$ direction, in our case). The sign of the power-flow direction of a wave can be computed with the following expression, related to the group velocity [2] (the slope of the $\beta-\omega$ dispersion diagram):

$$
\text { PowerFlowDirection }=\operatorname{sign}\left(\frac{\partial \beta_{y}}{\partial \omega}\right) \hat{y}
$$

From the frequency evolution shown in Figure 19, it is seen that as expected, zeros with numbers 2,4 , and 6 correspond to the progressive space-harmonic solution (the $+y$ direction), while zeros 1,3 , and 5 are the solution of the corresponding regressive counterpart. The student must fix his or her attention on the progressive set of space-harmonic solutions (zeros 2,4 , and 6). Some of them have a positive phase constant (mode 2 in Figure 18), while others have a negative phase constant (modes 4 and 6 in Figure 18). The sign of $\beta_{y}$ indicates the direction of the phase change (related to the phase velocity). It is said that a propagating wave is a forward wave if its group and phase velocities are parallel, while if they are anti-parallel, this wave is said to be a backward wave. Table 1 summarizes all these concepts for the studied zeros.

The existence of backward waves is a very interesting phenomenon, known for many years $[6,20,21]$, which now has resulted in much interest in the scientific community due to the attractive and practical applications of metamaterials [22]. The PAMELA tool helps to understand this interesting phenomenon of backward waves, and the application of backward leaky-wave modes to leaky-wave antennas [23-24]. As done for uniform open waveguides, the radiation region of a mode in an open periodic waveguide can be determined with the following equation, which is an extension of Equation (8) for the case in which negative phase constants are allowed to exist (space harmonics):

$$
-1<\frac{\beta_{y n}}{k_{0}}<+1
$$

This last expression is the key formula for radiation from open periodic structures. If any space harmonic $\left(\beta_{y n}\right)$ of a given solu- tion is in the radiation region, the mode of the periodic open waveguide will radiate. It will have a complex solution for the set of space harmonics, with an imaginary part $\left(\alpha_{y}\right)$ accounting for the radiation losses:

$$
k_{y n}=\beta_{y n}-j \alpha_{y}=\beta_{y 0}+n \frac{2 \pi}{P}-j \alpha_{y}
$$

Depending on the case, the radiating space harmonic can be a backward leaky wave $\left(-1<\beta_{y n} / k_{0}<0\right)$ or a forward leaky wave $\left(0<\beta_{y / 1} / k_{0}<+1\right)$. Equation (12) can also be extended to the radiation of any space harmonic, obtaining its elevation angle of maximum radiation:

$$
\sin \theta_{m, n} \cong \frac{\beta_{y n}}{k_{0}}
$$

The user must obtain the frequency-dispersion response for the leaky-wave mode using the zero with number 2 in Figure 18. This zero was associated with the main progressive space harmonic of the open periodic waveguide. The program automatically detects that the structure is periodic $(P \neq Q)$ and obtains the set of space harmonics associated with this solution, using Equation (19). Figure 20 shows the results obtained for the phase constants of each space harmonic and the angle of radiation, Equation (20), for a frequency sweep from $25 \mathrm{GHz}$ to $65 \mathrm{GHz}$,

Some interesting results can be obtained from Figure 20 . The harmonic phase constants graph shows the evolution of $n=0,-1,-2$ space hamonics. The main space-harmonic curve resembles the dispersion of the phase constant of the waveguide mode, starting from close to zero near the cutoff frequency $(30 \mathrm{GHz}$ ), and increasing its value with the frequency (as it corresponds to a progressive wave, see Equation (17)). Therefore, the radiation region of this main space harmonic is always in the forward quadrant $\left(\beta_{0} / k_{0}>1\right)$. In contrast, the $n=-1$ space hamonic emerges from the backward-leaky zone at $38 \mathrm{GHz}$ $\left(\beta_{-1} / k_{0}>1 \rightarrow \theta_{m-1}<0^{\circ}\right)$ and crosses the forward-leaky region up to $58 \mathrm{GHz}$ frequency, where this harmonic converts to a surface wave. The second-order space harmonic behaves in a similar manner. The backward-to-forward scanning radiation mechanism of a periodic leaky-wave antenna is represented in Figure $21 \mathrm{a}$, and can be easily understood from these last results. As in the uniform leaky-wave antenna, the traveling-wave nature of the antenna provides a frequency-scanning behavior with frequency. The main difference is that the periodic perturbation allows excitation of the $n=-1$ harmonic, which can scan from backward to forward as the frequency is increased. The phase constant of the $n=-1$ harmonic

Table 1. Classification of the zeros found in Figure 18.

\begin{tabular}{|c|c|c|c|c|}
\hline $\begin{array}{c}\text { Zero } \\
\text { Number }\end{array}$ & $\begin{array}{c}\beta_{y} \text { Solution at 40 GHz } \\
(\mathbf{r a d} / \mathbf{m})\end{array}$ & $\begin{array}{c}\text { Harmonic Index } \\
n\end{array}$ & $\begin{array}{c}\text { Power Flow } \\
\text { Direction }\end{array}$ & $\begin{array}{c}\text { Phase Change } \\
\text { Direction }\end{array}$ \\
\hline 2 & +1275.2 & $n=0$ & Progressive, $+y$ & Forward, $+y$ \\
\hline 4 & -582.6 & $n=-1$ & Progressive, $+y$ & Backward, $-y$ \\
\hline 6 & -2433.9 & $n=-2$ & Progressive, $+y$ & Backward, $-y$ \\
\hline 1 & +2433.9 & $n=-2$ & Regressive, $-y$ & Backward, $+y$ \\
\hline 3 & +582.6 & $n=-1$ & Regressive, $-y$ & Backward, $+y$ \\
\hline 5 & -1275.2 & $n=0$ & Regressive, $-y$ & Forward, $-y$ \\
\hline
\end{tabular}


$\left(\beta_{y-1}\right)$ changes its sign from negative (backward) to positive (forward), but the power direction inside the guide is always positive $\left(\partial \beta_{y-1} / \partial \omega>0\right)$. However, the radiated beam is backward directed when the wave phase velocity is negative $\left(\beta_{y-1}<0\right)$, due to the Fourier transform of such a backward illumination. The objective is to design the open waveguide dimensions and the periodic perturbations to make the $n=-1$ space harmonic radiale in the desired frequency band.

However, there are some frequency bands in which the phase constant seems to vanish. These zones are called prohibited band gaps, and are common to all periodic structures, such as photonic crystals [25] or frequency-selective surfaces [26]. In these frequency bands, a reactive phenomenon occurs, therefore avoiding propagation along the periodic structure. This phenomenon can be a desired effect in some applications: for instance, in avoiding the excitation of surface waves in antenna applications, or when we want to reject the propagation of waves in some specified directions of the structure. However, in our case, the band gaps are an unwanted effect that does not allow the antenna to radiate at some specific frequencies.

In Figure 20 , a band gap appears at $44 \mathrm{GHz}$ in the broadside radiation direction of the $n=-1$ space harmonic. Another band gap is observed at $54 \mathrm{GHz}$. Figure 22 shows the value of the normalized attenuation constant, $\alpha_{y} / k_{0}$, of this leaky-wave mode. A rapid taise in the value of $\alpha_{y}$ at the aforementioned band-gap frequencies can be seen. As happened to leaky-wave modes below cutoff, this high value of $\alpha_{y}$ is not due to an increase in the radiation losses, but to a reactive rejection of the signal.
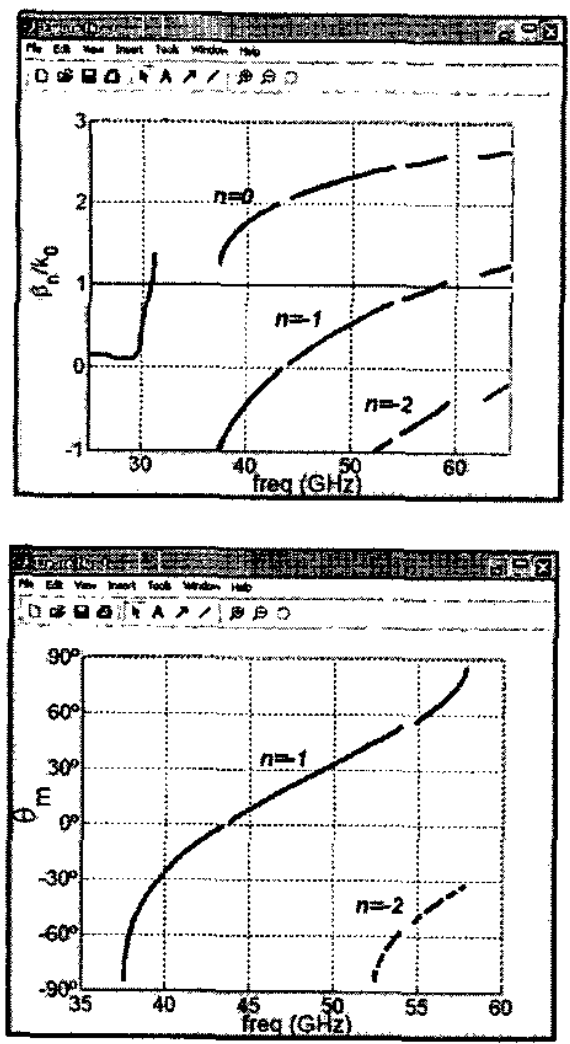

Figure 20. The frequency dispersion of the phase constant and radiation angle of the space harmonics.
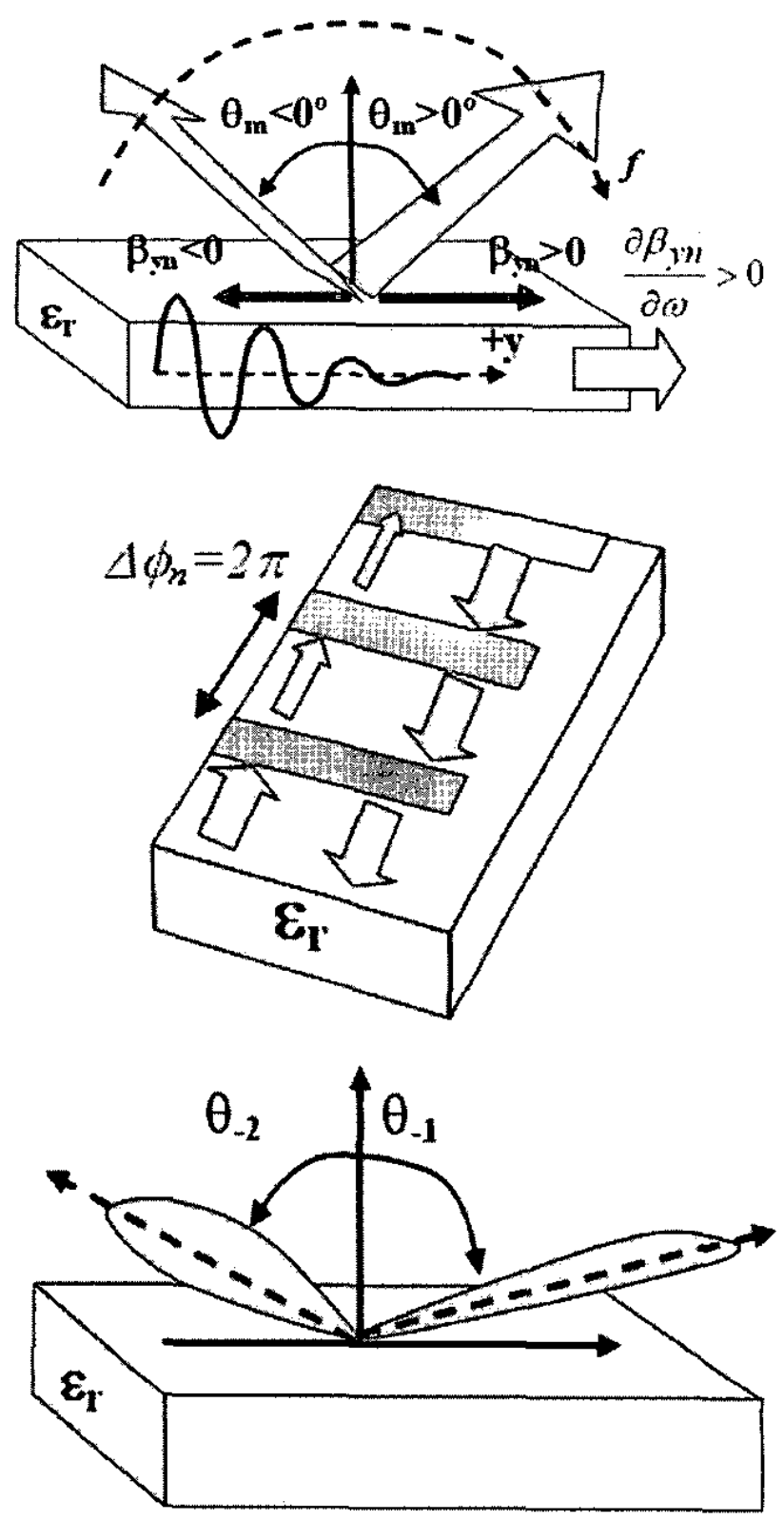

Figure 21. (a, top) The backward-to-forward scanning phenomenon. (b, middle) The spectral-gap phenomenon. (c, bottom) Multi-harmonic radiation.

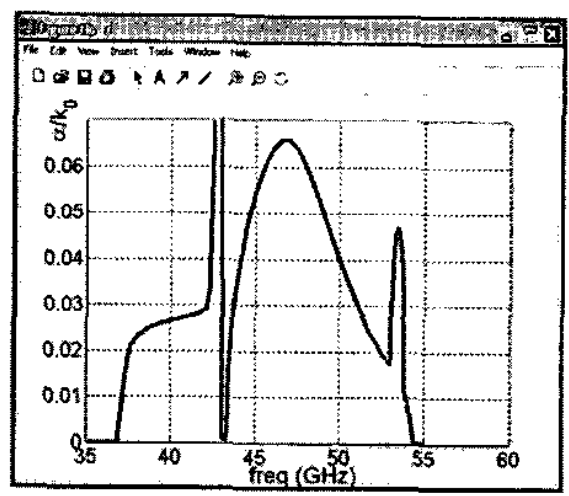

Figure 22. The normalized attenuation constant for the leakywave mode in an open periodic waveguide, showing the rapid rise of $\alpha_{y}$ in the bandgap regions. 


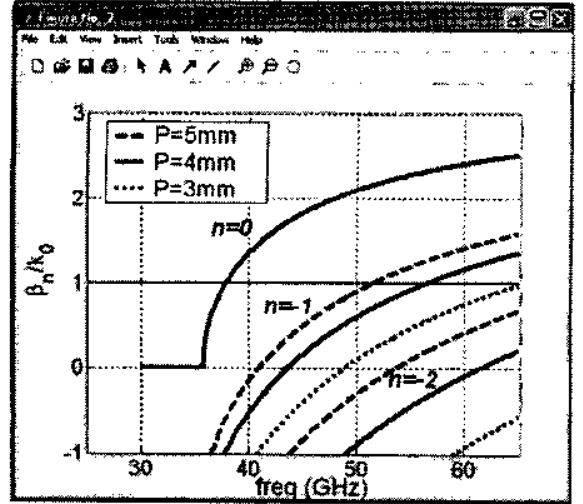

Figure 23. Space-harmonic curves obtained for different period lengths, $P$. The results were obtained for the unperturbed $\mathrm{TE}_{10}$ mode of the closed dielectric guide.

This reactive behavior of the wave can be explained by a simple destructive-interference phenomenon, illustrated in Figure 21b [27]. The progressive wave incident on the strip is partially reflected back towards the source at each strip. Due to the fact that the distance between adjacent strips is $P$, the phase difference of an $n$-order space harmonic, reflected back from a strip to the previous strip, can be computed as

$$
\Delta \phi_{n}=\beta_{n} P=\left(\beta_{0}+n \frac{2 \pi}{P}\right) P=\beta_{0} P+n 2 \pi \mathrm{rad}
$$

When $\Delta \phi$ is a multiple of $\pi$, the reflected waves from each strip add in-phase, creating a maximum of power reflected to the source, and therefore rapidly attenuating the resulting wave as it propagates along the structure in the progressive direction. The whole process is represented in Figure 21b. From Equation (21), it is easily shown that one of the band gaps occurs when the antenna is scanning at broadside $\left(\beta_{-1}=0\right)$, which corresponds to a value of $\beta_{0}=-2 \pi / P$. In this case, Equation (21) leads to a phase difference $\Delta \phi_{-1}=2 \pi$. The same result $\left(\Delta \phi_{n}=2 \pi\right)$ is obtained when any higher-order harmonic reaches the broadside direction. For this reason, this type of periodic leaky-wave antenna cannot scan in broadside. Some modifications must be made to allow radiation in this direction [27].

A last important phenomenon is observed from Figure 20. It can be seen how the $n=-1$ and the $n=-2$ harmonics radiate at the same time above $52 \mathrm{GHz}$. Each space harmonic radiates a main, narrow beam at its own elevation angle. This effect is illustrated in Figure 21c, and is not desirable in many applications, since the multi-harmonic radiation can produce unwanted interference. One important aspect in the design of a practical periodic leaky-wave antenna is to choose the period length $(P)$ in order to avoid radiation from several hamonics. To understand this idea, the student must have in mind Equation (14). For higher values of the period length, $P$, the spectral distance between consecutive harmonics is reduced. This can produce the result that a given higher-order harmonic reaches its radiation region (Equation (18)) before the lower-order harmonic has converted into a surface wave. In practice, this reasoning is applied for the $n=0$ and $n=-1$ harmonics. Figure 23 shows the results obtained by varying $P$ for a theoretical set of space harmonics in an ideal open rectangular waveguide, in which the main space hamonic would be obtained from the $\mathrm{TE}_{10}$ mode of the dielectric rectangular closed waveguide. In a real leaky-wave antenna design, the main space harmonic would be a perturbation of this $\mathrm{TE}_{10}$ mode. It can be seen that with $P=5 \mathrm{~mm}$, the $n=-1$ harmonic starts to radiate before the $n=0$ harmonic has left the radiation region $\left(\beta_{0} / k_{0}<1\right)$. As $P$ is decreased, the $n=-1$ harmonic moves away from the $n=0$ curve. The same process is applied to obtain the maximum scanning angle of the $n=-1$ hamonic, which is limited by the radiation of the $n=-2$ harmonic. However, the period, $P$, cannot be made as small as desired, since it can produce the result - that the first set of space harmonics (coming from the perturbed $\mathrm{TE}_{10}$ main mode) interfere with the next set of space harmonics (coming from the perturbed $\mathrm{TE}_{20}$ mode, for instance).

The main concepts that should have been leamed by the student in these last exercises are summarized in the following points:

1. Periodic guides can be analyzed by using the space-harmonic theory.

2. In the case of open periodic dielectric guides, any of the space harmonics must be in the radiation region to obtain a leaky-wave mode.

3. The main space harmonic $(n=0)$ can radiate only in the forward quadrant. The $n=-1$ harmonic can be used to frequency scan the radiated beam from backward to forward.

4. Prohibited band gaps occur at certain frequencies, in which the energy is reflected back to the source. One of these band gaps occurs at the broadside direction. "

5. Much care must be taken with simultaneous radiation from different harmonics, which turns into the appearance of radiation lobes at different elevation angles. The election of the correct period dimension is important to determine the maximum and minimum scan angles.

\section{Conclusions}

A software tool and an associated GUI environment has been developed for the analysis of the modal spectrum of laterallyshielded rectangular open waveguides, perturbed with planar metallizations. Due to its user-friendly interface, the input parameters can be easily introduced, and fast, visual results can be obtained in an interactive process. In this way, the user can learn many interesting concepts relating to leaky waves. The program permits analyzing a large variety of structures to obtain the allowed spectrum of modes, including leaky-wave modes in radiating structures. Several examples have been presented to aid in understanding the nature of leakage in different types of waveguides. First, radiation from uniform waveguides has been illustrated using a strip-loaded open dielectric rectangular waveguide. The surface-wave and the leaky-wave regimes have been studied, and the conditions for obtaining radiation have been checked, introducing the necessity for the excitation of the main parallel-plate mode in its "fast-wave" region. The cutoff regime for leaky waves has also been revised, illustrating the difference between radiation losses $\left(\alpha^{R A D}\right)$ and reactive losses for evanescent-cutoff complex waves $\left(\alpha^{R E A C T}\right)$. From the frequency-dispersion curves for both $\beta$ and $\alpha$, the radiation characteristics (the angle of maximum radiation, $\theta_{m}$, and 
the beamwidth, $\Delta \theta$ ) of a given leaky-wave mode were obtained. This showed the frequency-scanning capabilities of a uniform leaky-wave antenna, from near-broadside to end-fire.

The second basic type of leaky-wave antenna is the periodically perturbed open waveguide. The concept of space harmonics was introduced: it was shown that an infinite number of solutions can be found, all of them being related by the period of the structure. By properly choosing the operation frequency, it was shown that one can make the $n=-1$ space harmonic radiate from the backward direction to the forward direction. In this way, the student was also introduced to the interesting topic of left-handed propagation and metamaterials.

The software is being used in different courses at the Faculty of Telecommunication Engineering in Cartagena (Electromagnetic Field Laboratory second course, Microwave Engineering Laboratory fourth course, and Advanced Antenna Design course - $\mathrm{PhD}$ course). This tool has also been used at the Technical University of Valencia, as part of the invited $\mathrm{PhD}$ courses. To the authors" knowledge, this is the only tool specifically designed for helping in the teaching of leaky-wave modes. The results so far obtained (enquiries to the students, and exams) show that the method is succeeding in helping the teachers and students in the knowledge of the complex topics of leaky waves and backward radiation.

Readers can find a free, standalone, executable version of PAMELA at the following link:

http://www.tic.upct.es/html/investigacion/geat.

\section{References}

1. N. Marcuvitz, Waveguide Handbook, New York, McGraw-Hill, 1951.

2. R. E. Collin, Field Theory of Guided Waves, New York, McGraw-Hill, 1960.

3. T. Rozzi and M. Mongiardo, Open Electromagnetic Waveguides, London, IEE Electromagnetic Waves Series, 1997.

4. A. A. Oliner, "Physical Description of Parasitic Mode Effects and Their Influence on Crosstalk and Package Effects," Proceedings of Workshop on Loss, Crosstalk and Package Effects in Microwave and Millimeter-Wave Integrated Circuits, 1991 IEEE MTT-S Int. Microwave Symposium Digest.

5. H. Shigesawa and M. Tsuji, "Leaky-Wave Phenomena and Their Unfavorable Effect in Millimeter-Wave Circuit Devices," Microwave Conference, 2001 APMC, Volume 1, 2001, pp. 53-58.

6. A. A. Oliner, Leaky-wave antennas, in R. C. Johnson (ed.), Antenna Engineering Handbook. Third Edition, New York, McGraw-Hill, 1993, Chapter 10.

7. N. Marcuvitz, "On Field Representation in Terms of Leaky Modes," IRE Transactions on Antennas and Propagation, AP-4, 1956, pp. 192-194.

8. T. Tamir, "Inhomogeneous Waves Types at Planar Interfaces: III-Leaky Waves," OPTIK, 38, 3, February 1973, pp. 269-297.
9. W. Menzel, "A New Travelling-Wave Antenna in Microstrip," Arch. Elekr. Uebertrag Tech, 33, April 1979, pp. 137-140.

10. A. A. Oliner, "Leakage from Higher Modes on Microstrip Line with Application to Antennas," Radio Science, 22, 6, November 1987, pp. $907-912$

11. K. C. Gupta, T. Itoh, and A. A. Oliner, "Microwave and RF Education - Past, Present, and Future," IEEE Transactions on Microwave Theory and Techniques, MTT-50, 3, March 2002, pp. 1006-1014.

12. J. L. Gómez and A. A. Melcón, "Non-Orthogonality Relations Between Complex-Hybrid-Modes: An Application for the LeakyWave Analysis of Laterally-Shielded Top-Open Planar Transmission Lines," IEEE Transactions on Microwave Theory and Techniques, MTT-52, 3, March 2004, pp. 760-767.

13. J. L. Gómez and A. A. Melcón, "Radiation Analysis in the Space Domain of Laterally-Shielded Planar Transmission Lines. Part I: Theory," Radio Science, 39, 3, June 2004, pp. 1-11.

14. H. Shigesawa, M. Tsuji, P. Lampariello, F. Frezza and A. A. Oliner, "Coupling Between Different Leaky-Mode Types in StubLoaded Leaky Waveguides," IEEE Transactions on Microwave Theory and Techniques, 42, August 1994, pp. 1548-1560.

15. J. L. Gómez, A. A. Mejcón, and F. D. Quesada, "A Full-Wave Space-Domain Method for the Analysis of Leaky-Wave modes in Multilayered Planar Open Parallel-Plate Waveguides," International Journal of RF and Microwave Computer-Aided Engineering, 15, 1, January 2005, pp. 128-139.

16. P. Lampariello, F. Frezza and A. A. Oliner, "The Transition Region Between Bound-Wave and Leaky-Wave Ranges for a Partially Dielectric-Loaded Open Guiding Structure," IEEE Transactions on Microwave Theory and Techniques, MTT-38, 12, December 1990, pp. 1831-1836.

17. J. L. Gómez and A. A. Melcón, "Radiation Analysis in the Space Domain of Laterally-Shielded Planar Transmission Lines. Part II: Applications," Radio Science, 39, 3, June 2004, pp. 12-22.

18. J. L. Gómez, A. de la Torre, D. Cañete, M. Gugliemi and A. A. Melcón, "Design of Tapered Leaky-Wave Antennas in Hybrid Waveguide-Planar Technology for Millimeter Waveband Applications," IEEE Transactions on Antennas and Propagation, AP-53, 8, August 2005, pp. 2563-2577.

19. M. Guglielmi and G. Boccalone, "A Novel Theory for Dielectric-Inset Waveguide Leaky-Wave Antennas," IEEE Transactions on Antennas and Propagation, AP-49, 1991, pp. 497-504.

20. F. Schwering and S. T. Peng, "Design of Dielectric Grating Antennas for Millimeter-Wave Applications," IEEE Transactions on Antennas and Propagation, AP-31, February 1983, pp. 199. 209.

21. V. G. Veselago, "Electrodynamics of Substances with Simultaneously Negative Values of $\mathrm{mu}$ and epsilon," Soviet Physics Uspekhi, 10, January-February 1968, pp. 509-514.

22. D. R. Smith, W. J. Padilla, D. C. Vier, R. Shelby, S. C. NematNasser, N. Kroll, and S. Schultz, "Left Handed Metamaterials," 
Photonic Crystals and Light Focalization, Costas M. Soukoulis (ed.), Dordrecht, Kluwer Academic, 2001, p. 351.

23. A. Grbic and G. V. Eleftheriades, "Experimental Verification of Backward-Wave Radiation from a Negative Refractive Index Metamaterial," Joumal of Applied Physics, 92, November 2002, pp. 5930-5935.

24. L. Liu, C. Caloz, and T. Itoh, "Dominant Mode (DM) Leaky Wave Antenna with Backfire-to-Endfire Scanning Capability," Electron. Lett., 38, 23, November 2002, pp. 1414-1416.

25. L. Brillouin, Wave Propagation in Periodic Structures: Electric Filters and Crystal Lattices, New York, McGraw Hill, 1946; New York, Dover Publications, 1953.

26. B. A. Munk, Frequency Selective Surfaces: Theory and Design, New York, John Wiley \& Sons, 2000, ISBN 0-471-37047-9.

27. M. Guglielmi and D. R. Jackson, "Broadside Radiation from Periodic Leaky-Wave Antennas," IEEE Transactions on Antennas and Propagation, AP-41, 1, January 1993.

\section{Introducing the Feature Article Authors}

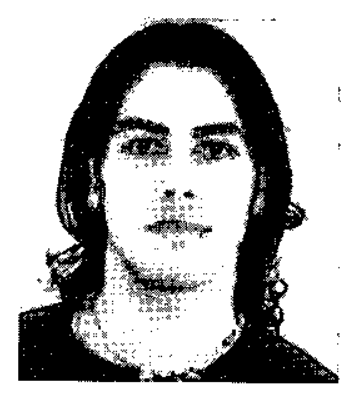

José Luis Gómez Tornero was born in Murcia, Spain, in 1977. He received the Telecommunications Engineer degree from the Polytechnic University of Valencia (UPV), Valencia, Spain, in 2001 , and the "laurea cum laude" $\mathrm{PhD}$ degree in Telecommunication Engineering from the Technical University of Cartagena (UPCT), Cartagena, Spain, in 2005.

In 1999, he joined the Radiocommunications Department, UPV, as a research student, where he was involved in the development of analytical and numerical tools for the study and automated design of microwave filters in waveguide technology for space applications. In 2000, he joined the Radio Frequency Division, Industry Alcatel Espacio, Madrid, Spain, where he was involved with the development of microwave active circuits for telemetry, tracking, and control (TTC) transponders involved in many different space missions for the European Space Agency (ESA), the National Aeronautics Space Administration (NASA), and other space agencies. In 2001, he joined the Technical University of Cartagena, Spain, as an Assistant Professor, where he is currently developing his teaching activities. His scientific research is focused on the analysis and design of leaky-wave antennas for millimeter-wave applications, and the development of numerical methods for the analysis of novel passive radiating structures in planar and waveguide technologies. His scientific interests also include the study of active devices for microwave and millimeter wave bands, such as oscillators and active antennas.
In July, 2004, José Luis Gómez the second national award from the foundation EPSON-Ibérica for the best $\mathrm{PhD}$ project in the field of Technology of Information and Communications (TIC). Since October, 2005, José Luis Gómez has occupied the position of Vice Dean for Students and Lecture Affairs in the Technical University of Cartagena.

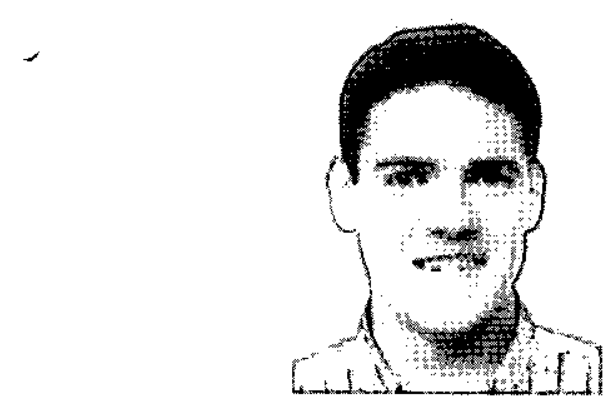

David Cañete Rebenaque was bom in Valencia, Spain, in 1976. He received the Telecommunications Engineer degree from the Technical University of Valencia, Valencia, Spain, in 2001, and he is currently working toward the $\mathrm{PhD}$ degree at the Technical University of Cartagena, Cartagena, Spain.

During 2001, he worked as an RF engineer for a mobile communication company. In 2002, he joined the Communications and Information Technologies Department, Technical University of Cartagena. His research interests include the analysis and design of microwave circuits and active antennas.

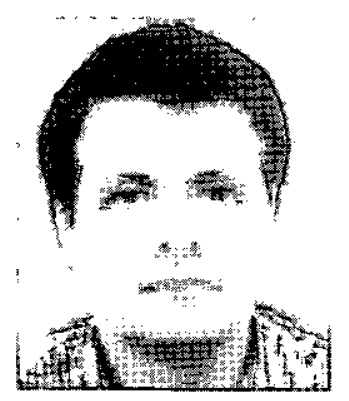

Alejandro Alvarez Melcón was born in Madrid, Spain, in 1965. He received the Telecommunications Engineer degree from the Polytechnic University of Madrid (UPM), Madrid, Spain, in 1991, and the $\mathrm{PhD}$ degree in Electrical Engineering from the Swiss Federal Institute of Technology, Lausanne, Switzerland, in 1998.

In 1988, he joined the Signal, Systems, and Radiocommunications Department, UPM, as a research student, where he was involved in the design, testing, and measurement of broadband spiral antennas for electromagnetic-measurement support (EMS) equipment. From 1991 to 1993, he joined the Radio Frequency Systems Division, European Space Agency (ESA/ESTEC), Noordwijk, The Netherlands, where he was involved in the development of analytical and numerical tools for the study of waveguide discontinuities, planar transmission lines, and microwave filters. From 1993 to 1995 , he joined the Space Division, Industry Alcatel Espacio, Madrid, Spain, and he worked at the ESA, where he collaborated in several ESA/ESTEC contracts. From 1995 to 1999 , he was with the Swiss Federal Institute of Technology, École Polytechnique Fédérale de Lausanne, 
Lausanne, Switzerland, where he worked in the field of microstrip antennas and printed circuits for space applications. In 2000, he joined the Technical University of Cartagena, Spain, where he is currently developing his teaching and research activities.

Dr. Alejandro Alvarez Melcón received the JNNA (Journée Internationales de Nice sur les Antennes) Best Paper Award for the best contribution to the JINA'98 International Symposium on Antennas, and the COIT/AEIT (Colegio Oficial de Ingenieros de Telecomunicación) award for the best $\mathrm{PhD}$ thesis in basic information and communication technologies.

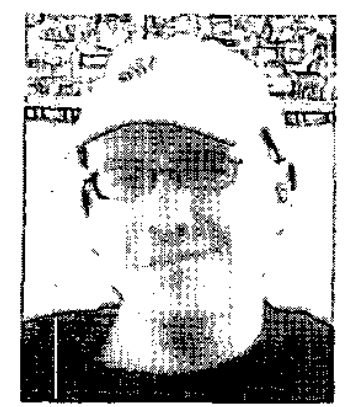

Fernando Quesada Pereira was bom in Murcia, Spain, in 1974. He received the Telecommunications Engineer degree from the Technical University of Valencia (UPV), Valencia, Spain, in 2000 , and is currently working toward the $\mathrm{PhD}$ degree at the Technical University of Cartagena (UPCT), Cartagena, Spain. In 1999, he joined the Radiocommunications Department, UPV, as a Research Assistant, where he was involved in the development of numerical methods for the analysis of anechoic chambers and tag antennas. In 2001, he joined the Communications and Information Technologies Department, UPCT, initially as a Research Assistant, and then as an Assistant Professor. In 2005, he spent six months as a Visiting Scientist with the University of Pavia, Pavia, Italy. His current scientific interests include integral equation numerical methods for the analysis of antennas and microwave devices.

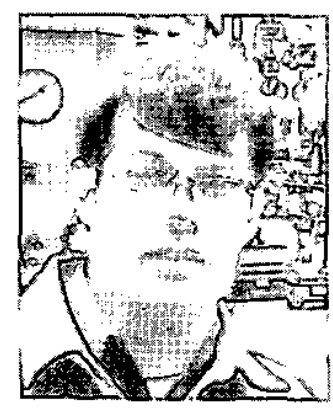

Juan Pascual Gareía was born in Castellón, Spain, in 1975. He received the Telecommunications Engineer degree from the Technical University of Valencia, Valencia, Spain, in 2001, and is currently working toward the $\mathrm{PhD}$ degree at the Technical University of Cartagena (UPCT), Cartagena, Spain. In 2002, he joined the CEMI Research Center, UPCT, as a Research Assistant. In 2003, he joined the Communications and Information Technologies Department, UPCT. His research interests include neural networks, genetic algorithms, and their applications in the analysis and development of a CAD tool for microwave circuits and antennas, (niti) 